\title{
Projective klt pairs with nef anti-canonical divisor
}

\author{
Frédéric Campana, Junyan Cao and Shin-ichi Matsumura
}

\begin{abstract}
In this paper, we study projective klt pairs $(X, \Delta)$ with nef anti-log canonical divisor $-\left(K_{X}+\Delta\right)$ and their maximal rationally connected fibration $\psi: X \rightarrow Y$. We prove that the numerical dimension of $-\left(K_{X}+\Delta\right)$ on $X$ coincides with that of $-\left(K_{X_{y}}+\Delta_{X_{y}}\right)$ on a general fiber $X_{y}$ of $\psi: X \rightarrow Y$, which is an analogue of Ejiri-Gongyo's result formulated for the Kodaira dimension. As a corollary, we obtain a relation between the positivity of the anti-canonical divisor and the rational connectedness, which provides a sharper estimate than that in Hacon-McKernan's question. Moreover, in the case of $X$ being smooth, we show that $X$ admits a "holomorphic" maximal rationally connected fibration to a smooth projective variety $Y$ with numerically trivial canonical divisor, and also that this is locally trivial with respect to the pair $(X, \Delta)$, which generalizes Cao-Höring's structure theorem to the case of klt pairs. Finally, we consider slope rationally connected quotients of $(X, \Delta)$ and obtain a structure theorem for projective orbifold surfaces.
\end{abstract}

\section{Introduction}

The study of certain "positively curved" varieties, which are often formulated to have positive bisectional curvatures, tangent bundles, or anti-canonical divisors, occupies an important place in the theory of the classification of varieties. One of the central problems in this study is to determine the structure of semi-positively curved varieties in terms of naturally associated fibrations such as Albanese maps, Iitaka fibrations, or maximal rationally connected fibrations. Particularly, the study of nef anti-canonical divisors, which has been developed rapidly in recent decades, covers a large range of positively curved varieties and is thus extremely important.

Cao-Höring recently established a structure theorem for a smooth projective variety $X$ with nef anti-canonical divisor (see [CH19]): $X$ admits a locally trivial fibration $X \rightarrow Y$ to a smooth projective variety $Y$ with numerically trivial canonical divisor such that the fiber is rationally connected. By combining this with the Beauville-Bogomolov decomposition (see [Bea83]), the uni-

Received 30 January 2020, accepted in final form 6 January 2021.

2020 Mathematics Subject Classification 14D06 (primary), 14E30, 32J25 (secondary).

Keywords: klt pairs, nef anti-canonical divisors, Beauville-Bogomolov decomposition, rational connectedness, MRC fibrations, positivity of direct image sheaves, singular Hermitian metrics, numerical dimension, numerical flatness, slope rationally connected quotients.

This journal is (C) Foundation Compositio Mathematica 2021. This article is distributed with Open Access under the terms of the Creative Commons Attribution Non-Commercial License, which permits non-commercial reuse, distribution, and reproduction in any medium, provided that the original work is properly cited. For commercial re-use, please contact the Foundation Compositio Mathematica.

The third author is supported by Grant-in-Aid for Young Scientists (A) $\sharp 17$ H04821, Grant-in-Aid for Scientific Research (B) $\sharp 21 H 00976$, Fostering Joint International Research (A) $\sharp 19 K K 0342$ from JSPS, and the JSPS Program for Advancing Strategic International Networks to Accelerate the Circulation of Talented Researchers. This work is partially supported by the Agence Nationale de la Recherche through a "Convergence de Gromov-Hausdorff en géométrie kählérienne" (ANR-GRACK) grant. 


\section{PROJECTIVE KLT PAIRS WITH NEF ANTI-CANONICAL DIVISOR}

versal cover of $X$ can be decomposed into the product of $\mathbb{C}^{m}$, Calabi-Yau manifolds, hyperkähler manifolds, and rationally connected manifolds. Several important previous studies include [CP91, DPS93, DPS94, DPS96, Zha96, Pău97, CDP15, Cao19, CH19] and the references therein.

This paper is devoted to the study of projective klt pairs $(X, \Delta)$ with nef anti-log canonical divisor $-\left(K_{X}+\Delta\right)$. It is natural and of interest to consider log pairs rather than varieties themselves from the viewpoint of birational geometry. We focus on structures of maximal rationally connected (MRC) fibrations and slope rationally connected (sRC) quotients, as introduced in [Cam92, KMM92] and [Cam17], respectively. This paper contains three main results: The first result is a more general solution to the question posed by Hacon-M $\mathrm{M}^{\mathrm{C}}$ Kernan in [HM07] (see Theorem 1.2). The second result is a generalization of [CH19] to projective klt pairs (see Theorem 1.3). The third result contributes to a structure of sRC quotients and provides a structure theorem for projective orbifold surfaces (see Theorem 1.6).

\subsection{Positivity of anti-canonical divisors - Hacon-McKernan's question}

The following question, posed by Hacon- $\mathrm{M}^{\mathrm{c}}$ Kernan, concerns a relation between the rational connectedness of $X$ and the positivity of $-\left(K_{X}+\Delta\right)$.

Problem 1.1 (Hacon-McKernan's question, [HM07, Question 3.1]). Let $(X, \Delta)$ be a projective klt pair with nef anti-log canonical divisor $-\left(K_{X}+\Delta\right)$, and let $\psi: X \rightarrow Y$ be an MRC fibration of $X$. Then, does the following inequality hold?

$$
\kappa\left(-\left(K_{X}+\Delta\right)\right) \leqslant \operatorname{dim} X-\operatorname{dim} Y,
$$

where $\kappa(\bullet)$ is the Kodaira dimension.

This question can be seen as a generalization of a fundamental result in birational geometry; weak Fano varieties are rationally connected. Indeed, the condition $\operatorname{dim} Y=0$ is equivalent to the rational connectedness of $X$, and thus the right-hand side in the question measures how far $X$ is from rationally connected varieties.

Ejiri-Gongyo in [EG19] affirmatively solved the above question by proving the following inequality:

$$
\kappa\left(-\left(K_{X}+\Delta\right)\right) \leqslant \kappa\left(-\left(K_{X_{y}}+\Delta_{X_{y}}\right)\right),
$$

where $X_{y}$ is a general fiber of an MRC fibration $\psi: X \rightarrow Y$ and $K_{X_{y}}+\Delta_{X_{y}}$ is the log canonical divisor defined by the restriction to $X_{y}$. In this paper, we prove an analogue of Ejiri-Gongyo's result by replacing the Kodaira dimension with the numerical dimension. Moreover, as an application, we resolve Hacon-McKernan's question in the following strong form.

ThEOREM 1.2. Let $(X, \Delta)$ be a projective klt pair with nef anti-log canonical divisor $-\left(K_{X}+\Delta\right)$, and let $\psi: X \rightarrow Y$ be an MRC fibration of $X$. Then, the following equality holds:

$$
\operatorname{nd}\left(-\left(K_{X}+\Delta\right)\right)=\operatorname{nd}\left(-\left(K_{X_{y}}+\Delta_{X_{y}}\right)\right)
$$

for a general fiber $X_{y}$ of $\psi$. Here $\mathrm{nd}(\bullet)$ is the numerical dimension and $-\left(K_{X_{y}}+\Delta_{X_{y}}\right)$ is the anti-log canonical divisor on $X_{y}$. In particular, we obtain a generalization of Hacon- $M^{c}$ Kernan's question:

$$
\operatorname{nd}\left(-\left(K_{X}+\Delta\right)\right) \leqslant \operatorname{dim} X-\operatorname{dim} Y
$$

We emphasize that there are several advantages in considering the numerical dimension instead of the Kodaira dimension. For example, the equality in Theorem 1.2 does not hold without 


\section{F. Campana, J. Cao and S. Matsumura}

considering the numerical dimension, which is one of the interesting points. Indeed, there is a counterexample to the equality for the Kodaira dimension, although Ejiri-Gongyo's inequality holds (see Example 2.11). Moreover, our inequality in Theorem 1.2 not only recovers Hacon$M^{c}$ Kernan's question but also gives a sharper estimate by the well-known formula $\kappa(\bullet) \leqslant \operatorname{nd}(\bullet)$.

The inequality nd $\left(-\left(K_{X}+\Delta\right)\right) \geqslant \operatorname{nd}\left(-\left(K_{X_{y}}+\Delta_{X_{y}}\right)\right)$ in Theorem 1.2 is proved by an extension theorem of holomorphic sections from a general fiber $X_{y}$ to the ambient space $X$. On the other hand, the converse inequality requires a deeper insight into the direct image sheaf of the plurianti-canonical divisor $-m\left(K_{X}+\Delta\right)$. The key points in this step are to demonstrate a certain flatness of the direct image sheaf and to estimate the dimension of global sections and the rank of flat vector bundles.

\subsection{Structure theorem of MRC fibrations for klt pairs}

From the viewpoint of birational geometry, it is of interest to ask whether Cao-Höring's structure theorem can be extended to log pairs. This question is answered in a quite general form (see Theorem 4.3), which is one of the contributions of this paper. Moreover, by considering in detail the case where $X$ is smooth, we generalize Cao-Höring's structure theorem to klt pairs, as follows.

Theorem 1.3. Let $(X, \Delta)$ be a projective klt pair such that $X$ is smooth and the anti-log canonical divisor $-\left(K_{X}+\Delta\right)$ is nef. Then, there exists a (holomorphic) MRC fibration $\psi: X \rightarrow Y$ with the following properties:

(1) The variety $Y$ is smooth and $c_{1}(Y)=0$.

(2) The fibration $\psi$ is locally trivial with respect to $(X, \Delta)$, namely, for any small (Euclidean) open set $U \subset Y$, there is the isomorphism

$$
\left(\psi^{-1}(U), \Delta\right) \cong U \times\left(X_{y}, \Delta_{X_{y}}\right)
$$

over $U \subset Y$, where $X_{y}$ is the fiber of $\psi$.

Moreover, using the Beauville-Bogomolov decomposition, the universal cover of $X$ can be decomposed into the product of $\mathbb{C}^{m}$, Calabi-Yau manifolds, hyperkähler manifolds, and rationally connected manifolds.

Theorem 1.3 provides a powerful tool for understanding the geometry of nef anti-canonical divisors. For example, Ejiri-Gongyo's inequality, explained in Subsection 1.1, can be easily reproved from the above theorem. Indeed, for an effective $\mathbb{Q}$-divisor $D$ with $D \sim_{\mathbb{Q}}-\left(K_{X}+\Delta\right)$, the pair $(X, \Delta+\delta D)$ is still klt and has nef anti-log canonical divisor for sufficiently small $\delta>0$. Then, since $D$ is $\psi$-horizontal by Theorem 1.3, the restriction map

$$
H^{0}\left(X,-m\left(K_{X}+\Delta\right)\right) \longrightarrow H^{0}\left(X_{y},-m\left(K_{X_{y}}+\Delta_{X_{y}}\right)\right)
$$

is injective, which leads to Ejiri-Gongyo's inequality.

The proof of Theorems 4.3 and 1.3 gives a generalization of the argument in [CH19] to log pairs and also contains several new ingredients in the generalized setting (see Setting 3.1), for example, Propositions 2.6 and 2.7, Theorems 4.3 and 1.3, and Subsection 4.3.

\subsection{Structure theorem of SRC quotients for orbifolds}

In the latter part of this paper, we begin to study the structure of sRC quotients. The sRC quotient, introduced in [Cam17], is a fibration naturally associated with $\log$ pairs $(X, D)$ and can 


\section{ProjeCtive KLT PAIRS WITH NEF ANTI-CANONICAL DIVISOR}

be seen as a generalization of MRC fibrations. The MRC fibration is defined for $X$, whereas the sRC quotient is defined for log pairs $(X, D)$.

Let us briefly review the definition of sRC quotients, following [Cam17]. An orbifold morphism $f:\left(X, D_{X}\right) \rightarrow\left(Y, D_{Y}\right)$ between $\log$ pairs $\left(X, D_{X}\right)$ and $\left(Y, D_{Y}\right)$ is a morphism $f: X \rightarrow Y$ such that $f(X)$ is not contained in $D_{Y}$ and that

$$
c_{f^{\star}(E)}(F) \cdot \frac{1}{1-c_{D_{X}}(F)} \geqslant \frac{1}{1-c_{D_{Y}}(E)}
$$

holds for any irreducible component $E$ of $D_{Y}$ and irreducible component $F$ of $f^{\star} E$. Here $c_{D}(G)$ denotes the multiplicity of a $\mathbb{Q}$-divisor $D$ in a prime divisor $G$. The notion of rationally connected manifolds can be generalized to log canonical (lc) pairs $(X, D)$ with smooth $X$ as follows: The pair $(X, D)$ is said to be $s R C$ if for any ample line bundle $A$ on $X$, there exists a positive integer $m_{A}$ such that

$$
\left.h^{0}\left(X^{\prime},\left(\otimes^{m} \pi^{\star} \Omega^{1}(X, D)\right) \otimes \pi^{\star} A\right)\right)=0 \quad \text { for any } m \geqslant m_{A},
$$

where $\pi: X^{\prime} \rightarrow(X, D)$ is some (or any) Kawamata cover adapted to $(X, D)$. See [CP19, Definition 5.3] for the definition and basic properties of the orbifold cotangent bundle $\pi^{\star} \Omega^{1}(X, D)$. The following theorem defines sRC quotients.

Theorem 1.4 ([Cam17, Theorem 1.5, Corollary 10.6]). Let $(X, D)$ be a lc pair with smooth $X$. Then, there exist an orbifold birational model $\left(X^{\prime}, D^{\prime}\right)$ of $(X, D)$ and an orbifold morphism $\rho:\left(X^{\prime}, D^{\prime}\right) \rightarrow\left(R, D_{R}\right)$ onto a smooth orbifold $\left(R, D_{R}\right)$ with the following properties:

(1) The general orbifold fibers $\left(X_{r}, D_{r}\right)$ are $s R C$.

(2) The divisor $K_{R}+D_{R}$ is pseudo-effective.

Here $\left(X_{r}, D_{r}\right)$ is a general orbifold fiber (that is, the pair of a general fiber $X_{r}$ and $\left.\left.D\right|_{X_{r}}\right)$. Such a fibration is uniquely determined up to orbifold birational equivalence.

Moreover, in the case where $-\left(K_{X}+D\right)$ is nef, the orbifold base $\left(R, D_{R}\right)$ satisfies

$$
\kappa\left(K_{R}+D_{R}\right)=\operatorname{nd}\left(K_{R}+D_{R}\right)=0 .
$$

The orbifold morphism $\rho:\left(X^{\prime}, D^{\prime}\right) \rightarrow\left(R, D_{R}\right)$ in the above theorem is called a $s R C$ quotient of $(X, D)$. If an orbifold pair $(X, D)$ is sRC, then $X$ itself is rationally connected. However, the converse implication does not hold when $D$ is a nontrivial divisor. This means that the sRC quotient of $(X, D)$ might not be trivial, even if the MRC fibration of $X$ is trivial. Therefore, we can extract more information from the sRC quotients than from MRC fibrations alone.

It is natural to ask whether a structure theorem for sRC quotients can be obtained in the same spirit as Theorem 1.3. Motivated by this question, we pose the following conjecture.

Conjecture 1.5. Let $(X, D)$ be a klt pair such that $X$ is smooth and $-\left(K_{X}+D\right)$ is nef. Then, there exists an orbifold morphism $\rho:(X, D) \rightarrow\left(R, D_{R}\right)$ with the following properties:

(1) The pair $\left(R, D_{R}\right)$ is a klt pair such that $R$ is smooth and $c_{1}\left(K_{R}+D_{R}\right)=0$.

(2) The general orbifold fibers $\left(X_{r}, D_{r}\right)$ are sRC.

(3) The morphism $\rho$ is locally trivial with respect to the pairs; namely, for any small (Euclidean) open set $U \subset R$, there is the isomorphism

$$
\left(\rho^{-1}(U), D\right) \cong\left(U, D_{R}\right) \times\left(X_{r}, D_{r}\right)
$$

over $U \subset Y$, where $X_{r}$ is the fiber of $\rho$. 


\section{F. Campana, J. Cao and S. Matsumura}

In this paper, we prove the conjecture in the case where $(X, D)$ is a smooth orbifold surface (see Theorem 1.6). Moreover, as a step toward proving Conjecture 1.5 in its full generality, we prove a certain birational semistability theorem (see Theorem 5.11).

Theorem 1.6. Let $(X, D)$ be a $\log$ smooth klt pair such that $X$ is a surface and $-\left(K_{X}+D\right)$ is nef. Then, Conjecture 1.5 holds; that is, there exists an orbifold morphism $\rho:(X, D) \rightarrow\left(R, D_{R}\right)$ satisfying the conclusions of Conjecture 1.5.

Moreover, if $(X, D)$ is not $s R C$ and $K_{X}+D$ is not numerically trivial, then $\rho:(X, D) \rightarrow$ $\left(R, D_{R}\right)$ is nontrivial and there exists an effective $\mathbb{Q}$-divisor $D_{\mathbb{P}^{1}}$ on $\mathbb{P}^{1}$ satisfying the following:

- We have $\operatorname{deg} D_{\mathbb{P}^{1}}<2$.

- The curve $R$ is either $\mathbb{P}^{1}$, if $h^{1}\left(X, \mathcal{O}_{X}\right)=0$, or else an elliptic curve.

- We have $(X, D) \cong\left(R, D_{R}\right) \times\left(\mathbb{P}^{1}, D_{\mathbb{P}^{1}}\right)$ locally over the base $R$ and globally if $h^{1}\left(X, \mathcal{O}_{X}\right)=0$.

The second part of Theorem 1.6 is stronger than Conjecture 1.5. The sRC quotient in the theorem gives a more precise description than the classical MRC fibration (which is trivial), except when $(X, D)$ is sRC or when $-\left(K_{X}+D\right)$ is numerically trivial. Later in this paper, we show that Conjecture 1.5 cannot be expected for (non-klt) lc pairs (see Example 5.10), which is one of our contributions.

The remainder of this paper is organized as follows: Section 2 develops the theory of the positivity of direct image sheaves and numerically flat vector bundles. In Section 3, as a step toward the proof of Theorem 4.3, we generalize the key propositions obtained in [Cao19] and [CH19] to almost holomorphic maps with nef "relative" anti-canonical divisor. In Section 4, we prove Theorems 1.2, 4.3, and 1.3 by applying the theory established in Sections 2 and 3. In Section 5, we discuss properties of sRC quotients for orbifolds with nef anti-log canonical divisor.

\section{Preliminaries}

In this section, we first summarize several results regarding the positivity of direct image sheaves and then develop the theory of numerically flat vector bundles. The new ingredients here are Propositions 2.6, 2.7, and 2.8.

\subsection{Positivity of direct image sheaves}

In this subsection, we briefly recall the notion of singular Hermitian metrics on vector bundles (more generally, torsion-free sheaves) and the theory of the positivity of direct image sheaves (see [Ber09, BP08, PT18, HPS18] for more details).

Let $E$ be a (holomorphic) vector bundle of rank $r$ on a complex manifold $Y$. A singular Hermitian metric $h$ on $E$ is a Hermitian metric that can be locally written as a measurable map with values in semi-positive Hermitian forms on $\mathbb{C}^{r}$ such that $0<\operatorname{det} h<+\infty$ almost everywhere. A singular Hermitian vector bundle $(E, h)$ is said to be positively curved if $\log |s|_{h^{\star}}$ is a plurisubharmonic (psh) function on $U$ for any open set $U \subset Y$ and any section $s \in H^{0}\left(U, E^{\star}\right)$, where $h^{\star}:={ }^{t} h^{-1}$ is the dual Hermitian metric of $h$ on the dual bundle $E^{\star}$. In the case where $h$ is a smooth Hermitian metric, $(E, h)$ is positively curved if and only if the Chern curvature associated with $h$ is semi-positive in the sense of Griffith. For a smooth $(1,1)$-form $\theta$ on an open set $U \subset Y$, we simply write

$$
\sqrt{-1} \Theta_{h}(E) \succeq \theta \otimes \operatorname{id}_{E} \quad \text { on } U
$$




\section{PROJECTIVE KLT PAIRS WITH NEF ANTI-CANONICAL DIVISOR}

if $d d^{c} \log |s|_{h^{\star}}-\theta$ is semi-positive in the sense of currents for any open set $U^{\prime} \subset U$ and any section $s \in H^{0}\left(U^{\prime}, E^{\star}\right)$. The notation $\sqrt{-1} \Theta_{h}(E)$ is used in the above definition, but an appropriate definition of the curvature is, in general, unknown for singular Hermitian metrics. We now introduce the notion of weakly positively curved torsion-free sheaves.

Definition 2.1. Let $E$ be a torsion-free coherent sheaf on a complex manifold $Y$ and $\omega_{Y}$ be a fixed Hermitian form on $Y$. The sheaf $E$ is said to be weakly positively curved if for any real number $\varepsilon>0$, there exists a singular Hermitian metric $h_{\varepsilon}$ on $\left.E\right|_{Y_{1}}$ such that

$$
\sqrt{-1} \Theta_{h_{\varepsilon}}(E) \succeq-\varepsilon \omega_{Y} \otimes \operatorname{id}_{E} \quad \text { on } Y_{1},
$$

where $Y_{1}$ is the maximal locally free locus of $E$.

For a proper morphism $\varphi: \Gamma \rightarrow Y$ with connected fibers, the direct image sheaf of the plurirelative canonical bundle $m K_{\Gamma / Y}$ twisted by a line bundle $L$, that is, $\varphi_{\star}\left(m K_{\Gamma / Y}+L\right)$, admits a positively curved singular Hermitian metric under suitable assumptions; this comes from the fundamental works developed by several authors, including Berndtsson, Păun, and Takayama (see, for example, [Ber09, BP08, PT18] and the references therein). The results on the positivity of direct image sheaves, which play an important role in this paper, can be summarized in the following form. The first conclusion in the following theorem is derived from [BP08, CP17, PT18]. The second conclusion is an application of the first. Here, we only provide a proof for the second conclusion.

Theorem 2.2 ([CP17, Lemma 5.25], [PT18, BP08]). Let $\varphi: \Gamma \rightarrow Y$ be a surjective morphism with connected fibers between smooth projective varieties and $(L, h)$ be a singular Hermitian line bundle on $\Gamma$. Let $\theta$ be a smooth $(1,1)$-form on $Y$ and $m$ be a positive integer such that

$$
\sqrt{-1} \Theta_{h}(L) \geqslant \varphi^{\star} \theta \text { and } \mathcal{I}\left(\left.h^{1 / m}\right|_{\Gamma_{y}}\right)=\mathcal{O}_{\Gamma_{y}},
$$

where $\Gamma_{y}$ is a general fiber. The following hold:

(1) The induced singular Hermitian metric $H$ on the direct image sheaf $\varphi_{\star}\left(m K_{\Gamma / Y}+L\right)$ satisfies

$$
\sqrt{-1} \Theta_{H}\left(\varphi_{\star}\left(m K_{\Gamma / Y}+L\right)\right) \succeq \theta \otimes \operatorname{id}_{Y} \quad \text { on } Y_{1} \text {. }
$$

(2) Let $N$ be a nef line bundle on $\Gamma$. We further assume that $L$ is $\varphi$-big. Then, for every $\varepsilon>0$, there exists a singular Hermitian metric $H_{\varepsilon}$ on the direct image sheaf $\varphi_{\star}\left(m K_{\Gamma / Y}+N+L\right)$ that satisfies

$$
\sqrt{-1} \Theta_{H_{\varepsilon}}\left(\varphi_{\star}\left(m K_{\Gamma / Y}+N+L\right)\right) \succeq\left(\theta-\varepsilon \omega_{Y}\right) \otimes \operatorname{id}_{Y} \quad \text { on } Y_{1} .
$$

In particular, in the case $\theta=0$, the direct image sheaf $\varphi_{\star}\left(m K_{\Gamma / Y}+N+L\right)$ is weakly positively curved.

Here $Y_{1}$ is the maximal locally free locus of the direct image sheaves.

Proof of Theorem 2.2(2). There exist a singular Hermitian metric $H$ on $L$ and a smooth Hermitian metric $g_{\delta}$ on $N$ such that

$$
\sqrt{-1} \Theta_{H}(L)+\varphi^{\star} \omega_{Y} \geqslant \omega \text { and } \sqrt{-1} \Theta_{g_{\delta}}(N) \geqslant-\delta \omega
$$

for some Kähler form $\omega_{Y}$ on $Y$ and some Kähler form $\omega$ on $\Gamma$, because $L$ is $\varphi$-big and $N$ is nef. Then, the metric $h_{\varepsilon}$ on $L+N$ defined by $h_{\varepsilon}:=h^{1-\varepsilon} H^{\varepsilon} g_{\varepsilon}$ satisfies

$$
\mathcal{I}\left(\left.h_{\varepsilon}^{1 / m}\right|_{\Gamma_{y}}\right)=\mathcal{I}\left(\left.h^{(1-\varepsilon) / m} H^{\varepsilon / m}\right|_{\Gamma_{y}}\right)=\mathcal{O}_{\Gamma_{y}} \text { and } \sqrt{-1} \Theta_{h_{\varepsilon}}(L+N) \geqslant \varphi^{\star}\left(\theta-\varepsilon \omega_{Y}\right)
$$

for any $1 \gg \varepsilon \gg \delta>0$. Therefore, item (2) follows from item (1). 


\section{F. Campana, J. Cao and S. Matsumura}

\subsection{Numerically flat vector bundles}

In this subsection, we first show that if a reflexive sheaf $\mathcal{F}$ is weakly positively curved and satisfies $c_{1}(\mathcal{F})=0$, then $\mathcal{F}$ is actually a vector bundle and is numerically flat. This was first proved in [CH19, Corollary 2.12] for projective surfaces. We generalize [CH19, Corollary 2.12] to arbitrary dimension in virtue of Bando-Siu's result for admissible Hermitian-Einstein metrics (see [BS94]). Note that this result has been slightly generalized to pseudo-effective reflexive sheaves (see [HIM21]) using the same method as in this paper and has been generalized to the Kähler setting (see [Wu20]). There is also a geometric approach in [GKP16, HP19] for a more general setting.

We first recall the definition of numerically flat vector bundles and the fundamental work of [DPS94] on a characterization in terms of filtrations of Hermitian flat vector bundles.

Definition 2.3. Let $E$ be a vector bundle on a compact complex manifold $Y$. The vector bundle $E$ is said to be numerically flat if $E$ is nef and its dual bundle $E^{\star}$ is also nef; equivalently, $E$ is a nef vector bundle satisfying

$$
c_{1}(E)=c_{1}(\operatorname{det} E)=0 \in H^{1,1}(Y, \mathbb{R}) .
$$

Here $E$ is said to be nef if the tautological line bundle $\mathcal{O}_{\mathbb{P}(E)}(1)$ is nef on the projective space bundle $\mathbb{P}(E)$.

TheOREM 2.4 ([DPS94]). Let $E$ be a vector bundle on a compact Kähler manifold $Y$. Then, the following conditions are equivalent:

- The vector bundle $E$ is numerically flat.

- There exists a filtration of $E$ by subbundles

$$
0=: E_{0} \subset E_{1} \subset \cdots \subset E_{p-1} \subset E_{p}:=E
$$

such that each quotient bundle $E_{k} / E_{k-1}$ is a Hermitian flat vector bundle for any $1 \leqslant$ $k \leqslant p$ (that is, the quotient $E_{k} / E_{k-1}$ admits a smooth Hermitian metric $h_{k}$ such that $\left.\sqrt{-1} \Theta_{h_{k}}\left(E_{k} / E_{k-1}\right)=0\right)$.

The following lemma, which is needed for the proof of Proposition 2.6, follows by induction on the dimension, the local cohomology, and the standard argument of restriction to hypersurfaces.

LEMMA 2.5. Let $E$ be a vector bundle on a smooth projective variety $Y$ and $Y_{1}$ be a Zariski open set in $Y$ with $\operatorname{codim}\left(Y \backslash Y_{1}\right) \geqslant 2+i$. Then, the morphism

$$
H^{j}(Y, E) \longrightarrow H^{j}\left(Y_{1}, E\right)
$$

induced by the restriction is an isomorphism for any $j \leqslant i$.

Proof. The proof is done by induction on the dimension of $Y$. Let $Z$ be the reduced subvariety defined by the complement $Z:=Y \backslash Y_{1}$ and $H$ be a general hypersurface in the complete linear system of a very ample line bundle on $Y$. By the induction hypothesis, the morphism

$$
H^{j}\left(H, \mathcal{O}_{H}(E)\right) \longrightarrow H^{j}\left(H_{1}, \mathcal{O}_{H_{1}}(E)\right)
$$

is an isomorphism for any $j \leqslant i$, where $H_{1}:=H \backslash(Z \cap H)$. Taking $H$ to be sufficiently ample, we may assume that

$$
h^{k}\left(Y, \mathcal{O}_{Y}\left(E \otimes H^{\star}\right)\right)=h^{n-k}\left(Y, \mathcal{O}_{Y}\left(K_{Y} \otimes E^{\star} \otimes H\right)\right)=0
$$




\section{PROJECTIVE KLT PAIRS WITH NEF ANTI-CANONICAL DIVISOR}

for any $k<\operatorname{dim} Y$. Let us consider the long exact sequence on $Y$ induced by

$$
0 \longrightarrow \mathcal{O}_{Y}\left(E \otimes H^{\star}\right) \longrightarrow \mathcal{O}_{Y}(E) \rightarrow \mathcal{O}_{H}(E) \longrightarrow 0
$$

and its restriction to $Y_{1}$. Then, from the vanishing result (2.1) and the induction hypothesis, we see that it suffices to show that $H^{j}\left(Y_{1}, \mathcal{O}_{Y_{1}}\left(E \otimes H^{\star}\right)\right)=0$ for any $j \leqslant i$.

Using (2.1) again, we obtain

$$
H^{j}\left(Y_{1}, \mathcal{O}_{Y_{1}}\left(E \otimes H^{\star}\right)\right) \cong H_{Z}^{j+1}\left(Y, \mathcal{O}_{Y}\left(E \otimes H^{\star}\right)\right)
$$

from the long exact sequence of the local cohomology (see [Har67, Corollary 1.9]). Let $Z_{k}$ be the nonreduced scheme with structure sheaf $\mathcal{O}_{Y} / \mathcal{I}_{Z}^{k}$, where $\mathcal{I}_{Z}$ is the ideal sheaf of $Z$. Then, by [Har67, Theorem 2.8], the local cohomology can be computed as follows:

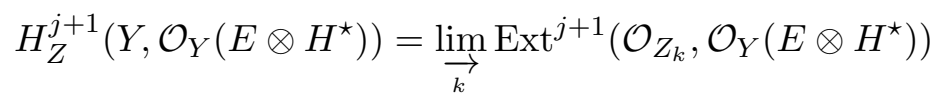

$$
\begin{aligned}
& =\underset{\vec{k}}{\lim _{\vec{m}}} \operatorname{Ext}^{j+1}\left(\mathcal{O}_{Z_{k}}\left(E^{\star} \otimes H \otimes K_{Y}\right), \mathcal{O}_{Y}\left(K_{Y}\right)\right) \text {. }
\end{aligned}
$$

The duality theorem implies that

$$
\begin{aligned}
& \operatorname{Ext}^{j+1}\left(\mathcal{O}_{Z_{k}}\left(E^{\star} \otimes H \otimes K_{Y}\right), \mathcal{O}_{Y}\left(K_{Y}\right)\right) \\
& \cong \text { the dual space of } H^{n-(j+1)}\left(Z_{k}, \mathcal{O}_{Z_{k}}\left(E^{\star} \otimes H \otimes K_{Y}\right)\right) .
\end{aligned}
$$

The right-hand side vanishes by the inequality $\operatorname{dim} Y-(j+1) \geqslant \operatorname{dim} Y-(i+1)>\operatorname{dim} Z$. This finishes the proof.

Proposition 2.6. Let $\mathcal{F}$ be a reflexive sheaf on a smooth projective variety $Y$. If $\mathcal{F}$ is weakly positively curved in the sense of Definition 2.1 and if we have $c_{1}(\mathcal{F})=0$, then $\mathcal{F}$ is a numerically flat vector bundle on $Y$.

Proof. The proof is done by induction on the rank of $\mathcal{F}$. Note that the conclusion is obvious in the case of rank 1 because any reflexive sheaves of rank 1 are invertible sheaves (see [Har80, Proposition 1.9]).

Let $Y_{1}$ be the maximal locally free locus of $\mathcal{F}$ and $A$ be an ample line bundle on $Y$. Note that $\operatorname{codim}\left(Y \backslash Y_{1}\right) \geqslant 3$ by the reflexivity of $\mathcal{F}$. The main idea of the proof is that, after taking an appropriate filtration of $\mathcal{F}$, we extend the subsheaves or quotient sheaves to vector bundles on $Y$ by applying the results of [CH19] and [BS94, Corollary 3].

First, we fix an ample line bundle $A$ on $Y$ and a coherent subsheaf $\mathcal{S} \subset \mathcal{F}$ with minimum rank among coherent subsheaves $\mathcal{S} \subset \mathcal{F}$ satisfying $\int_{Y} c_{1}(\mathcal{S}) \cdot c_{1}(A)^{n-1} \geqslant 0$. In the proof, we consider the exact sequence

$$
0 \longrightarrow \mathcal{S} \longrightarrow \mathcal{F} \longrightarrow \mathcal{Q}:=\mathcal{F} / \mathcal{S} \longrightarrow 0 \text {. }
$$

We may assume that $\mathcal{S}$ is reflexive and that the quotient sheaf $\mathcal{Q}=\mathcal{F} / \mathcal{S}$ is torsion-free by taking its saturation.

We now consider the case where $\operatorname{rank} \mathcal{S}=\operatorname{rank} \mathcal{F}$. In this case, we can see that $\mathcal{S}=\mathcal{F}$ and that there is no proper subsheaf $\mathcal{S}^{\prime}$ in $\mathcal{F}$ with $\int_{Y} c_{1}\left(\mathcal{S}^{\prime}\right) \cdot c_{1}(A)^{n-1} \geqslant 0$ by the construction of $\mathcal{S}$. This implies that the sheaf $\mathcal{F}$ is $A$-stable. Additionally, the restriction $\left.\mathcal{F}\right|_{S}$ to a surface $S$ is a pseudo-effective vector bundle by the inequality $\operatorname{codim}\left(Y \backslash Y_{1}\right) \geqslant 3$, where $S$ is a complete intersection

$$
S:=H_{1} \cap H_{2} \cap \cdots \cap H_{n-2}
$$




\section{F. Campana, J. Cao and S. Matsumura}

in $Y$ of general members $H_{i}$ of the complete linear system of $A$. Therefore, the vector bundle $\left.\mathcal{F}\right|_{S}$ is numerically flat on $S$, and thus we obtain $c_{2}\left(\left.\mathcal{F}\right|_{S}\right)=0$ (see [DPS94] or [CH19, Corollary 2.12]). This implies that

$$
\int_{Y} c_{2}(\mathcal{F}) \cdot c_{1}(A)^{n-2}=\int_{S} c_{2}\left(\left.\mathcal{F}\right|_{S}\right)=0
$$

Combining this with the assumption $c_{1}(\mathcal{F})=0$, we can conclude that $\mathcal{F}$ is a Hermitian flat vector bundle on $Y$, from the stability of $\mathcal{S}=\mathcal{F}$ (see [BS94, Corollary 3]). In particular, the sheaf $\mathcal{F}$ is locally free and numerically flat.

We consider the remaining case where $\operatorname{rank} \mathcal{S}<\operatorname{rank} \mathcal{F}$. As $\mathcal{F}$ is weakly positively curved, its quotient $\mathcal{Q}$ is also weakly positively curved. In particular, its first Chern class $c_{1}(\mathcal{Q})$ is pseudoeffective. Additionally, we have $0=c_{1}(\mathcal{F})=c_{1}(\mathcal{S})+c_{1}(\mathcal{Q})$ by the assumption on $\mathcal{F}$. Combining this with the choice of $\mathcal{S}$, we see that

$$
\int_{Y} c_{1}(\mathcal{S}) \cdot c_{1}(A)^{n-1}=0 \text { and } \int_{Y} c_{1}(\mathcal{Q}) \cdot c_{1}(A)^{n-1}=0 .
$$

We now show that $\mathcal{S}$ is a vector bundle on $Y_{1}$ and that the morphism $\mathcal{S} \rightarrow \mathcal{F}$ is a bundle morphism on $Y_{1}$, following the argument in [DPS94]. By the reflexivity of $\mathcal{F}$ and $\mathcal{S}$, it is sufficient to check that the induced morphism $\mathcal{Q}^{\star} \rightarrow \mathcal{F}^{\star}$ is actually injective as a bundle morphism on $Y_{1}$. Further, using [DPS94, Lemma 1.20], this problem can be reduced to checking that the induced morphism $\operatorname{det} \mathcal{Q}^{\star} \rightarrow \Lambda^{p} \mathcal{F}^{\star}$ is injective, where $p$ is the rank of $\mathcal{Q}$. This morphism determines the section

$$
\tau \in H^{0}\left(Y, \Lambda^{p} \mathcal{F}^{\star} \otimes \operatorname{det}\left(\mathcal{Q}^{\star}\right)^{\star}\right) .
$$

From $c_{1}(\mathcal{Q})=0$, we can infer that $\left(\operatorname{det} \mathcal{Q}^{\star}\right)^{\star}$ is a flat line bundle. Combining this with the assumption on $\mathcal{F}$, we see that $\Lambda^{p} \mathcal{F} \otimes \operatorname{det}\left(\mathcal{Q}^{\star}\right)$ is weakly positively curved. Then, we can show that $\tau$ is nonvanishing on $Y_{1}$ by the same argument as in [DPS94, Proposition 1.16]. This implies that $\mathcal{S}$ can be seen as a subbundle of $\mathcal{F}$ via the morphism $\mathcal{S} \rightarrow \mathcal{F}$ on $Y_{1}$. In particular, the quotient $\mathcal{Q}$ is also a vector bundle on $Y_{1}$.

We now have the surjective bundle morphism

$$
\Lambda^{r-s+1} \mathcal{F} \otimes \operatorname{det} \mathcal{Q}^{\star} \longrightarrow \mathcal{S}
$$

on $Y_{1}$, where $r$ and $s$ are the ranks of $\mathcal{F}$ and $\mathcal{S}$, respectively. The sheaf $\Lambda^{r-s+1} \mathcal{F} \otimes \operatorname{det} \mathcal{Q}^{\star}$ is weakly positively curved by the equality $c_{1}(\mathcal{Q})=0$ and the assumption on $\mathcal{F}$. Then, the induced metric on $\mathcal{S}$ is weakly positively curved on $Y_{1}$, and thus is so on $Y$ by the inequality $\operatorname{codim}\left(Y \backslash Y_{1}\right) \geqslant 3$. Therefore, $\mathcal{S}$ is a numerically flat vector bundle on $Y$ by the induction hypothesis. In particular, we obtain $c_{1}(\mathcal{S})=0$ and $c_{1}(\mathcal{Q})=0$. The sheaf $\mathcal{Q}$ is weakly positively curved, and thus so is the reflexive hull $\mathcal{Q}^{\star \star}$. Hence $\mathcal{Q}^{\star \star}$ is a numerically flat vector bundle on $Y$ by the equality $c_{1}(\mathcal{Q})=0$ and the induction hypothesis.

Finally, we prove that $\mathcal{F}$ is a vector bundle on $Y$. By the above argument, sequence (2.2) is an exact sequence of vector bundles on $Y_{1}$ and the quotient $\mathcal{Q}$ can be extended to a vector bundle $\mathcal{Q}^{\star \star}$ on $Y$. Note that $\mathcal{Q}$ itself cannot be expected to be a vector bundle on $Y$ because $\mathcal{Q}$ may not be reflexive. Applying Lemma 2.5 to our case, we see that the restriction map

$$
H^{1}\left(Y, \mathcal{Q}^{\star} \otimes \mathcal{S}\right) \longrightarrow H^{1}\left(Y_{1}, \mathcal{Q}^{\star} \otimes \mathcal{S}\right)
$$

is an isomorphism by the inequality $\operatorname{codim}\left(Y \backslash Y_{1}\right) \geqslant 3$. Let us consider the extension class in $H^{1}\left(Y_{1}, \mathcal{Q}^{\star} \otimes \mathcal{S}\right)$ obtained from the exact sequence (2.2) of vector bundles on $Y_{1}$. This extension class can be extended to that in $H^{1}\left(Y, \mathcal{Q}^{\star} \otimes \mathcal{S}\right)$. By construction, the extended class determines the vector bundle whose restriction to $Y_{1}$ corresponds to $\mathcal{F}$. This vector bundle coincides with $\mathcal{F}$ 


\section{PROJECTIVE KLT PAIRS WITH NEF ANTI-CANONICAL DIVISOR}

on $Y$ because $\mathcal{F}$ is reflexive (see [Har80, Proposition 16]). It then follows that $\mathcal{F}$ is nef since $\mathcal{S}$ and $\mathcal{Q}$ are nef.

Using the notion of numerical flatness, we obtain a criterion for local triviality with respect to log pairs with horizontal divisors. In general, for a surjective morphism $p: X \rightarrow Y$ between algebraic varieties $X$ and $Y$, a divisor $D$ on $X$ is said to be $p$-horizontal (respectively, $p$-vertical) if any component of $D$ is dominant over $Y$ (respectively, $D$ is not dominant over $Y$ ). The following criterion can be regarded as a pair version of [Cao19, Proposition 2.8]. For convenience and completeness, we briefly recall the argument in [Cao19] and present a precise proof.

Proposition 2.7 (cf. [Cao19, Proposition 2.8]). Let $p: X \rightarrow Y$ be a surjective morphism with connected fibers between compact Kähler manifolds, and let $\Delta$ be an effective reduced $p$-horizontal divisor on $X$. Let $L$ be a $p$-ample line bundle on $X$. We assume that the direct image sheaves

$$
p_{\star}(m L) \quad \text { and } \quad p_{\star}\left(m L \otimes \mathcal{I}_{\Delta}\right)
$$

are numerically flat vector bundles on $Y$ for $m$ sufficiently large. Then $p: X \rightarrow Y$ is locally trivial with respect to the pair $(X, \Delta)$. Moreover, for the universal cover $\pi: \tilde{Y} \rightarrow Y$ of $Y$, the fiber product $(\tilde{X}, \tilde{\Delta}):=(X, \Delta) \times_{Y} \tilde{Y}$ admits a splitting

$$
(\tilde{X}, \tilde{\Delta}) \cong \tilde{Y} \times\left(F,\left.\Delta\right|_{F}\right)
$$

where $F$ is the fiber of $p$.

Proof. We may assume that $L$ is $p$-very ample and the direct image sheaves in the proposition are numerically flat for any $m \geqslant 1$. We put $E_{m}:=p_{\star}(m L)$. By the $p$-relative embedding associated with $L$, we have the following $p$-relative embedding:

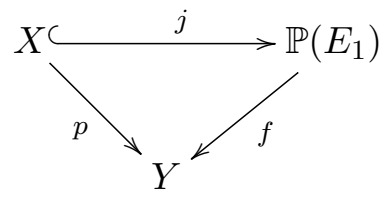

and $L=j^{\star} \mathcal{O}_{\mathbb{P}\left(E_{1}\right)}(1)$. Then, for $m$ sufficiently large, we have the exact sequence

$$
0 \rightarrow f_{\star}\left(\mathcal{O}_{\mathbb{P}\left(E_{1}\right)}(m) \otimes \mathcal{I}_{X}\right) \rightarrow f_{\star}\left(\mathcal{O}_{\mathbb{P}\left(E_{1}\right)}(m)\right) \rightarrow p_{\star}(m L) \rightarrow 0,
$$

where $\mathcal{I}_{X}$ is the ideal sheaf defined by the subvariety $X \subset \mathbb{P}\left(E_{1}\right)$. The vector bundle $E_{1}$ is determined by a local system since $E_{1}$ is numerically flat. Let $D_{E_{1}}$ be the flat connection with respect to this local system. As $\tilde{Y}$ is simply connected, the pull-back $\pi^{\star} E_{1}$ is a trivial vector bundle on $\tilde{Y}$, and thus we can take global flat sections (with respect to $D_{E_{1}}$ )

$$
\left\{e_{1}, e_{2}, \ldots, e_{r}\right\} \subset H^{0}\left(\tilde{Y}, \pi^{\star} E_{1}\right)
$$

generating $\pi^{\star} E_{1}$, where $r:=\operatorname{rank} E_{1}$.

The sheaf $F_{m}:=f_{\star}\left(\mathcal{O}_{\mathbb{P}\left(E_{1}\right)}(m) \otimes \mathcal{I}_{X}\right)$ is also a numerically flat locally free sheaf because both $f_{\star}\left(\mathcal{O}_{\mathbb{P}\left(E_{1}\right)}(m)\right)=\operatorname{Sym}^{m} E_{1}$ and $p_{\star}(m L)$ are numerically flat by the assumptions. In the same way as above, we see that the vector bundle $F_{m}$ admits a flat connection $D_{F_{m}}$ and $\pi^{\star} F_{m}$ is a trivial vector bundle. Hence we can take global flat sections (with respect to $D_{F_{m}}$ )

$$
\left\{s_{1}, s_{2}, \ldots, s_{t}\right\} \subset H^{0}\left(\tilde{Y}, \pi^{\star} F_{m}\right)
$$

generating $\pi^{\star} F_{m}$, where $t$ is the rank of $F_{m}$. 


\section{F. Campana, J. Cao and S. Matsumura}

Let us consider the injective morphism

$$
i: \pi^{\star} F_{m}=\pi^{\star} f_{\star}\left(\mathcal{I}_{X} \otimes \mathcal{O}_{\mathbb{P}\left(E_{1}\right)}(m)\right) \longrightarrow \pi^{\star} f_{\star}\left(\mathcal{O}_{\mathbb{P}\left(E_{1}\right)}(m)\right)=\pi^{\star} \operatorname{Sym}^{m} E_{1}
$$

induced by the exact sequence (2.3). Let $D$ be the flat connection on $\pi^{\star} \operatorname{Sym}^{m} E_{1}$ induced by $D_{E_{1}}$. Then, by [Cao13, Lemma 4.3.3], the section $i\left(s_{k}\right)$ is flat with respect to the connection $D$. In particular, the section $i\left(s_{k}\right)$ can be written as

$$
i\left(s_{k}\right)=\sum_{\alpha=\left(\alpha_{1}, \ldots, \alpha_{r}\right),|\alpha|=m} a_{k, \alpha} \cdot e_{1}^{\alpha_{1}} e_{2}^{\alpha_{2}} \cdots e_{r}^{\alpha_{r}}
$$

for some constants $a_{k, \alpha} \in \mathbb{C}$. The $\tilde{p}$-relative embedding of $\tilde{X}$ into $\mathbb{P}^{t-1} \times \tilde{Y}$ in the diagram

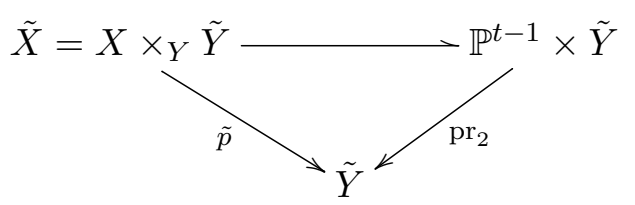

can be defined by the polynomials $i\left(s_{k}\right)$, whose coefficients are independent of $y \in \tilde{Y}$. This implies that $p$ is locally trivial and $\tilde{X}$ splits into $\tilde{X} \simeq \tilde{Y} \times F$, where $F$ is a general fiber of $p$.

We finally prove the local triviality with respect to the pair $(X, \Delta)$. We consider the restrictions $p_{\Delta}: \Delta \rightarrow Y$ of $p$ and $f_{\Delta}: \Delta \rightarrow Y$ of $f$ to $\Delta$. Further, we use the same notation $\mathcal{I}_{\Delta}$ to denote the ideal sheaf of $\Delta$ in both $X$ and $\mathbb{P}\left(E_{1}\right)$. For $m$ sufficiently large, we have the exact sequence

$$
0 \longrightarrow p_{\star}\left(m L \otimes \mathcal{I}_{\Delta}\right) \longrightarrow p_{\star}(m L) \longrightarrow p_{\Delta_{\star}}\left(m L \otimes \mathcal{O}_{X} / \mathcal{I}_{\Delta}\right) \longrightarrow 0
$$

As the first two terms are numerically flat, so is the third term $p_{\Delta_{\star}}\left(m L \otimes \mathcal{O}_{X} / \mathcal{I}_{\Delta}\right)$. By taking $m$ to be sufficiently large, we have another exact sequence

$$
0 \longrightarrow f_{\star}\left(\mathcal{O}_{\mathbb{P}\left(E_{1}\right)}(m) \otimes \mathcal{I}_{\Delta}\right) \longrightarrow f_{\star}\left(\mathcal{O}_{\mathbb{P}\left(E_{1}\right)}(m) \longrightarrow f_{\Delta_{\star}}\left(\mathcal{O}_{\mathbb{P}\left(E_{1}\right)}(m) \otimes \mathcal{O}_{\mathbb{P}\left(E_{1}\right)} / \mathcal{I}_{\Delta}\right) \longrightarrow 0 .\right.
$$

Then, since $\Delta$ is reduced, we see that

$$
f_{\Delta \star}\left(\mathcal{O}_{\mathbb{P}\left(E_{1}\right)}(m) \otimes \mathcal{O}_{\mathbb{P}\left(E_{1}\right)} / \mathcal{I}_{\Delta}\right)=p_{\Delta \star}\left(m L \otimes \mathcal{O}_{X} / \mathcal{I}_{\Delta}\right)
$$

is also numerically flat. Hence we can conclude that the first term $f_{\star}\left(\mathcal{I}_{\Delta} \otimes \mathcal{O}_{\mathbb{P}\left(E_{1}\right)}(m)\right)$ is numerically flat by $f_{\star}\left(\mathcal{O}_{\mathbb{P}\left(E_{1}\right)}(m)\right)=\operatorname{Sym}^{m} E_{1}$.

Applying the same argument as in the first part for $f_{\star}\left(\mathcal{O}_{\mathbb{P}\left(E_{1}\right)}(m) \otimes \mathcal{I}_{\Delta}\right)$, we can show that the $\tilde{p}$-relative embedding of $\tilde{\Delta}$ in $\mathbb{P}^{t-1} \times \tilde{Y}$ is defined by polynomials whose coefficients are independent of $y \in \tilde{Y}$. Hence we obtain the local triviality with respect to the pair $(X, \Delta)$ and a splitting $(\tilde{X}, \tilde{\Delta}) \cong \tilde{Y} \times\left(F,\left.\Delta\right|_{F}\right)$.

\subsection{Global sections of flat vector bundles}

In this subsection, we prove the following proposition, which is needed to estimate the dimension of global sections of flat vector bundles in the proof of Theorem 1.2.

Proposition 2.8. Let $\pi: \Gamma \rightarrow X$ be a birational morphism from a smooth projective variety $\Gamma$ to a (not necessarily smooth) projective variety $X$ and $\mathcal{F}$ be a torsion-free sheaf on $\Gamma$. Let $C$ be a complete intersection

$$
C:=H_{1} \cap H_{2} \cap \cdots \cap H_{\operatorname{dim} X-1}
$$

of a general member $H_{i}$ in the free linear system $\left|\pi^{\star} A\right|$, where $A$ is a very ample line bundle on $X$. Assume that the restriction $\left.\mathcal{F}\right|_{C}$ to a general complete intersection $C$ is a numerically flat 


\section{Projective KLT PAIRS WITH NEF ANTI-CANONICAL DIVISOR}

vector bundle on $C$. Then, for any line bundle $L$ on $\Gamma$, there exists a constant $C_{L}$ depending only on $L$ and $X$ (independent of $\mathcal{F}$ ) such that

$$
h^{0}(\Gamma, \mathcal{F} \otimes L) \leqslant C_{L} \cdot \operatorname{rank} \mathcal{F} .
$$

Before proving Proposition 2.8, we first consider the following lemma, which can be regarded as a vanishing theorem of Fujita type for numerically flat vector bundles.

Lemma 2.9. Let $A$ be an ample line bundle on a smooth projective variety $X$ and $E$ be a numerically flat vector bundle on $X$. Then, we have

$$
H^{q}\left(X, K_{X} \otimes E \otimes A\right)=0
$$

for any $q>0$.

Remark 2.10. It is natural to ask whether the same conclusion holds under the weaker assumption that $E$ is nef because it is true in the case where $E$ is a line bundle. When $X$ is a smooth curve, we can easily solve this question. Indeed, there is a finite morphism $p: X^{\prime} \rightarrow X$ such that $p^{\star}(E \otimes A)$ is the quotient of the direct sum $\oplus_{i} B_{i}$ of ample line bundles $B_{i}$ because $E \otimes A$ is an ample vector bundle (see [CF90]). Then, we see that

$$
\begin{aligned}
0 & =h^{1}\left(X^{\prime}, K_{X^{\prime}} \otimes p^{\star}(E \otimes A)\right)=h^{0}\left(X^{\prime}, p^{\star}\left(E^{\star} \otimes A^{\star}\right)\right) \\
& =h^{0}\left(X, E^{\star} \otimes A^{\star} \otimes p_{\star} \mathcal{O}_{X^{\prime}}\right) \geqslant h^{0}\left(X, E^{\star} \otimes A^{\star}\right) \\
& \geqslant h^{1}\left(X, K_{X} \otimes E \otimes A\right)
\end{aligned}
$$

by Serre duality, the projection formula, and the injectivity of $\mathcal{O}_{X} \hookrightarrow p_{\star} \mathcal{O}_{X^{\prime}}$.

Proof of Lemma 2.9. First, we observe that the conclusion is obvious if $E$ is a Hermitian flat vector bundle (that is, it admits a smooth Hermitian metric such that $\sqrt{-1} \Theta_{h}(E)=0$ ). Indeed, the vector bundle $E \otimes A$ is Nakano positive because the metric $h \otimes h_{A}$ satisfies

$$
\sqrt{-1} \Theta_{h \otimes h_{A}}(E \otimes A)=\mathrm{id}_{E} \otimes \sqrt{-1} \Theta_{h}(A)>_{\mathrm{Nak}} 0,
$$

where $h_{A}$ is a smooth Hermitian metric on $A$ with positive curvature. Therefore, in the case of $E$ being Hermitian flat, we obtain the desired conclusion from the Nakano vanishing theorem.

We now treat the general case of $E$ being numerically flat. Then, by Theorem 2.4, there exists a filtration of $E$ by subbundles $\left\{E_{k}\right\}_{k=0}^{p}$, that is,

$$
0=: E_{0} \subset E_{1} \subset \cdots \subset E_{p-1} \subset E_{p}:=E,
$$

such that the quotient bundle $E_{k} / E_{k-1}$ is a Hermitian flat vector bundle. The first observation yields $H^{q}\left(X, K_{X} \otimes E_{k} / E_{k-1} \otimes A\right)=0$ for any $q \geqslant 1$ and $1 \leqslant k \leqslant p$. Hence, we deduce

$$
h^{q}\left(X, K_{X} \otimes E_{k} \otimes A\right) \leqslant h^{q}\left(X, K_{X} \otimes E_{k-1} \otimes A\right)
$$

by using the long exact sequence obtained from the exact sequence

$$
0 \longrightarrow K_{X} \otimes E_{k-1} \otimes A \longrightarrow K_{X} \otimes E_{k} \otimes A \longrightarrow K_{X} \otimes E_{k} / E_{k-1} \otimes A \longrightarrow 0 .
$$

The above inequalities and the Hermitian flatness of $E_{1}$ yield

$$
h^{q}\left(X, K_{X} \otimes E \otimes A\right)=h^{q}\left(X, K_{X} \otimes E_{p} \otimes A\right) \leqslant h^{q}\left(X, K_{X} \otimes E_{1} \otimes A\right)=0 .
$$

This is the desired conclusion.

Proof of Proposition 2.8. For a given line bundle $L$ on $\Gamma$, we take a sufficiently ample line bundle $B$ on $X$ such that $L^{\star} \otimes \pi^{\star} B$ is a big line bundle on $\Gamma$. First, we show that

$$
H^{0}\left(\Gamma, \mathcal{F} \otimes L \otimes \pi^{\star} B^{\star}\right)=0
$$




\section{F. Campana, J. Cao and S. Matsumura}

by contradiction. Let $H$ be a general hyperplane in the free linear system $\left|\pi^{\star} A\right|$ and $t$ be a nonzero section

$$
t \in H^{0}\left(\Gamma, \mathcal{F} \otimes L \otimes \pi^{\star} B^{\star}\right) .
$$

Then the restriction $\left.t\right|_{H}$ is a nonzero section of $\left.\mathcal{F} \otimes L \otimes \pi^{\star} B^{\star}\right|_{H}$ and the restriction $\left.L^{\star} \otimes \pi^{\star} B\right|_{H}$ is a big line bundle on $H$. By repeating this process for general hypersurfaces in $\left|\pi^{\star} A\right|$, we can construct a smooth curve $C$ with the following properties:

- The curve $C$ is a complete intersection constructed by general members in $\left|\pi^{\star} A\right|$.

- The restriction $\left.t\right|_{C}$ is a nonzero section of $\left.\mathcal{F} \otimes L \otimes \pi^{\star} B^{\star}\right|_{C}$.

- The line bundle $\left.L^{\star} \otimes \pi^{\star} B\right|_{C}$ is still big on $C$ (and thus, ample on $C$ ).

Then, by Lemma 2.9 and Serre duality, we have

$$
h^{0}\left(C,\left.\mathcal{F} \otimes L \otimes \pi^{\star} B^{\star}\right|_{C}\right)=h^{1}\left(C,\left.K_{C} \otimes\left(\left.\mathcal{F}\right|_{C}\right)^{\star} \otimes L^{\star} \otimes \pi^{\star} B\right|_{C}\right)=0 .
$$

Here, we have used the assumption that $\left.\mathcal{F}\right|_{C}$ is numerically flat. This contradicts the existence of the nonzero section $\left.t\right|_{C}$.

We now consider a general hypersurface $H$ in $\left|\pi^{\star} B\right|$. We have the exact sequence

$$
\left.0 \longrightarrow \mathcal{F} \otimes L \otimes \pi^{\star} B^{\star} \longrightarrow \mathcal{F} \otimes L \longrightarrow \mathcal{F} \otimes L\right|_{H} \longrightarrow 0
$$

since $H$ is general and $\mathcal{F}$ is torsion-free. By the above argument and the induced long exact sequence, we obtain $h^{0}(X, \mathcal{F} \otimes L) \leqslant h^{0}\left(H,\left.\mathcal{F} \otimes L\right|_{H}\right)$. We may assume that $\left.L^{\star} \otimes \pi^{\star} B\right|_{H}$ is a big line bundle on $H$, and thus, by the same argument as above, we obtain

$$
h^{0}(X, \mathcal{F} \otimes L) \leqslant h^{0}\left(H,\left.\mathcal{F} \otimes L\right|_{H}\right) \leqslant h^{0}\left(H \cap H^{\prime},\left.\mathcal{F} \otimes L\right|_{H \cap H^{\prime}}\right)
$$

for general hypersurfaces $H$ and $H^{\prime}$ in $\left|\pi^{\star} B\right|$. By repeating this process, we obtain

$$
h^{0}(X, \mathcal{F} \otimes L) \leqslant h^{0}\left(C,\left.\mathcal{F} \otimes L\right|_{C}\right) \leqslant h^{0}\left(\Sigma,\left.\mathcal{F} \otimes L\right|_{\Sigma}\right)=\sharp \Sigma \cdot \operatorname{rank} \mathcal{F} .
$$

Here, $C$ is a general complete intersection of members of $\left|\pi^{\star} B\right|$ and $\Sigma$ is the set of the reduced points defined by $C \cap H$, where $H$ is a general hypersurface in $\left|\pi^{\star} B\right|$. The cardinality $\sharp \Sigma$ of $\Sigma$ is equal to the self-intersection number of $\pi^{\star} B$ and thus depends only on $B$ (and not on $\mathcal{F}$ ). This completes the proof.

In the rest of this subsection, we observe the following famous example (see [DPS94, Example 1.7] for more details), which tells us that the equality in Theorem 1.2 does not hold for the Kodaira dimension, even when $X$ is smooth and $\Delta$ is the zero divisor.

Example 2.11. Let $Y$ be an elliptic curve and $E$ be the (uniquely determined) vector bundle $E$ on $Y$ having the nonsplitting exact sequence

$$
0 \longrightarrow \mathcal{O}_{Y} \longrightarrow E \longrightarrow \mathcal{O}_{Y} \longrightarrow 0 \text {. }
$$

We consider the projective space bundle $\varphi: X:=\mathbb{P}(E) \rightarrow Y$. Then, the anti-canonical divisor $-K_{X}$ is nef (but not semi-ample). Additionally, we see that

$$
\kappa\left(-K_{X}\right)=0<1=\kappa\left(-K_{X_{y}}\right) \text { and } \operatorname{nd}\left(-K_{X}\right)=\operatorname{nd}\left(-K_{X_{y}}\right)=1,
$$

where $X_{y}$ is a fiber of the MRC fibration $\varphi: X \rightarrow Y$. Moreover, we can show that $E$ is numerically flat but not Hermitian flat. In this example, the direct image sheaves $\mathcal{V}_{m}$ defined in Section 3 are calculated through

$$
\mathcal{V}_{m}=\varphi_{\star}\left(-m K_{X / Y}\right)=\varphi_{\star}\left(-m K_{X}\right)=\varphi_{\star} \mathcal{O}_{\mathbb{P}(E)}(2 m)=\mathrm{S}^{2 m} E
$$




\section{PROJECTIVE KLT PAIRS WITH NEF ANTI-CANONICAL DIVISOR}

This example demonstrates that we actually need to treat numerically flat (not only Hermitian flat) vector bundles.

\section{Positivity of direct images of nef relative anti-canonical divisors}

We first describe our setting in this section. In Section 4, we consider the special case in this setting, namely, the case where $(X, \Delta)$ is (a $\mathbb{Q}$-factorial model of) a klt pair with nef anti-log canonical divisor and $\psi: X \rightarrow Y$ is an MRC fibration of $X$.

Setting 3.1. Let $\psi: X \rightarrow Y$ be an almost holomorphic map from a $\mathbb{Q}$-factorial projective variety $X$ to a smooth projective variety $Y$, and let $\pi: \Gamma \rightarrow X$ be a resolution of its indeterminacy locus and the singularities of $X$ with the morphism $\varphi: \Gamma \rightarrow Y$ in the following diagram:

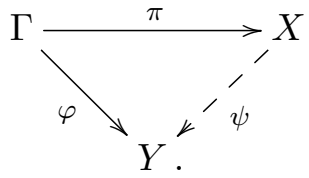

Assume that there exists an effective $\mathbb{Q}$-divisor $D$ on $\Gamma$ such that

$$
(\Gamma, D) \text { is klt and }-\pi_{\star}\left(K_{\Gamma / Y}+D\right) \text { is nef. }
$$

In this paper, for a $\mathbb{Q}$-divisor $L$ on $\Gamma$, the notation $\pi_{\star} L$ denotes the pushforward as Weil divisor. This assumption is equivalent to the existence of a $\pi$-exceptional $\mathbb{Q}$-divisor $E^{\prime \prime}$ on $\Gamma$ such that

$$
-\left(K_{\Gamma / Y}+D\right)+E^{\prime \prime} \text { is a nef } \mathbb{Q} \text {-divisor } .
$$

Let $E$ be the largest reduced $\pi$-exceptional divisor of $\pi: \Gamma \rightarrow X$. Note that the exceptional locus is divisorial since $X$ is $\mathbb{Q}$-factorial. When $X$ is smooth, the image $\varphi(E)$ is properly contained in $Y$. However, in general, it is possible that $\varphi(E)=\varphi\left(E^{\prime \prime}\right)=Y$. We choose a sufficiently ample line bundle $A$ on $\Gamma$ so that the natural morphism

$$
\operatorname{Sym}^{p} H^{0}\left(\Gamma_{y},\left.A\right|_{\Gamma_{y}}\right) \longrightarrow H^{0}\left(\Gamma_{y},\left.p A\right|_{\Gamma_{y}}\right)
$$

is surjective for any $p \in \mathbb{Z}_{+}$, where $\Gamma_{y}$ is a general fiber of $\varphi: \Gamma \rightarrow Y$. Let $Y_{0} \subset Y$ be the maximal Zariski open subset with the following properties:

- The induced morphism $\Gamma_{0}:=\varphi^{-1}\left(Y_{0}\right) \rightarrow Y_{0}$ has equidimensional fibers.

- The pull-back $\varphi^{\star} P$ is not contained in $E$ for any prime divisor $P \subset Y_{0}$.

Remark 3.2. (1) As pointed out in [Wan20, Remark 2.4], the draft of this paper did not take care of the $\mathbb{Q}$-factoriality of $X$. Some conclusions in this subsection hold without assuming the $\mathbb{Q}$ factoriality of $X$, but their proof will be much more technical and involved. To avoid unnecessary confusion, we assume that $X$ is $\mathbb{Q}$-factorial, but this setting is enough for our purposes. We emphasize that the main results in this paper can be proven without this assumption.

(2) Let $L$ be a $\mathbb{Q}$-divisor on $\Gamma$. Then, since $X$ is $\mathbb{Q}$-factorial, the pushforward $\pi_{\star} L$ as Weil divisor is actually $\mathbb{Q}$-Cartier and satisfies

$$
\mathcal{O}_{X}\left(p \pi_{\star} L\right) \cong\left(\pi_{\star} \mathcal{O}_{\Gamma}(p L)\right)^{\star \star}
$$

for $p$ divisible. In this paper, we interchangeably use the words "Cartier divisors," "invertible sheaves," and "line bundles," and so we simply denote the invertible sheaf $\mathcal{O}_{X}\left(p \pi_{\star} L\right)$ by $p \pi_{\star} L$.

Remark 3.3. For a given klt pair $(X, \Delta)$ with the nef anti-log canonical divisor $-\left(K_{X}+\Delta\right)$, we take an MRC fibration $\psi: X \rightarrow Y$ to a smooth variety $Y$ and its resolution $\pi: \Gamma \rightarrow X$ 


\section{F. Campana, J. Cao and S. Matsumura}

of its indeterminacies and the singularities of $X$, as in the diagram (3.1). Then Setting 3.1 is automatically satisfied except for the $\mathbb{Q}$-factoriality. Indeed, from [Zha05, Main theorem] and its proof, it follows that $\kappa(Y)=0$ and that $\varphi^{\star} N$ is $\pi$-exceptional for an effective $\mathbb{Q}$-divisor $N$ on $Y$ with $N \sim_{\mathbb{Q}} K_{Y}$. On the other hand, we have the formula of canonical divisors

$$
K_{\Gamma}+\Delta^{\prime}=\pi^{\star}\left(K_{X}+\Delta\right)+E^{\prime},
$$

where $\Delta^{\prime}$ and $E^{\prime}$ are effective $\mathbb{Q}$-divisors with no common components. By definition, the pair $\left(\Gamma, \Delta^{\prime}\right)$ is klt. Further, we obtain

$$
-\left(K_{\Gamma / Y}+\Delta^{\prime}\right) \sim_{\mathbb{Q}} \varphi^{\star} N-E^{\prime}-\pi^{\star}\left(K_{X}+\Delta\right) .
$$

The $\mathbb{Q}$-divisors $E^{\prime \prime}:=E^{\prime}-\varphi^{\star} N$ (which is $\pi$-exceptional) and $D:=\Delta^{\prime}$ satisfy the condition (3.3).

Our goal of this section is to prove Proposition 3.9 stating that appropriate direct image sheaves satisfy a certain flatness. For this purpose, we need some preliminary results.

The first lemma, which gives a certain birational semistability of $\psi: X \rightarrow Y$, was proved in [Zha05] when $\psi: X \rightarrow Y$ is an MRC fibration. The same proof still works in our setting.

Theorem 3.4 ([Zha05, Main theorem]). Under Setting 3.1, the following hold:

(1) Any irreducible component of $\pi\left(\varphi^{-1}\left(Y \backslash Y_{0}\right)\right)$ has codimension at least 2 . In particular, any $\varphi$-exceptional divisor is $\pi$-exceptional.

(2) Let $B$ be a prime divisor on $Y_{0}$ and $\varphi^{\star} B=\sum_{j} m_{j} B_{j}$ be the irreducible decomposition of the pull-back $\varphi^{\star} B$. If $B_{j}$ is a nonreduced component (that is, $m_{j}>1$ ), then $B_{j}$ is $\pi$-exceptional.

The following lemmas also play an important role in the proof; they follow from arguments similar to those used for [CH19, Lemmas 3.5 and 3.6].

Lemma 3.5. Let $L$ be a $\varphi$-big line bundle on $\Gamma$ and $P$ be a $\mathbb{Q}$-divisor on $Y$ such that $L-\varphi^{\star} P$ is pseudo-effective. Let $p \in \mathbb{Z}$ be an integer (which is not necessarily positive). If $\varphi_{\star}\left(p\left(K_{\Gamma / Y}+D\right)+L\right)$ is a nonzero sheaf, then

$$
\varphi_{\star}\left(p\left(K_{\Gamma / Y}+D\right)+L+c E^{\prime \prime}\right)-P
$$

is weakly positively curved on $Y$ for $c$ divisible and large with respect to $p$. This means that for any $\varepsilon>0$, there exists a singular Hermitian metric $h_{\varepsilon}$ such that

$$
\sqrt{-1} \Theta_{h_{\varepsilon}}\left(\varphi_{\star}\left(p\left(K_{\Gamma / Y}+D\right)+L+c E^{\prime \prime}\right)\right) \succeq\left(\theta-\varepsilon \omega_{Y}\right) \otimes \operatorname{id}_{Y},
$$

where $\theta$ is a smooth $(1,1)$-form on $Y$ representing $c_{1}(P)$. In particular, when $L$ is pseudo-effective, the direct image sheaf $\varphi_{\star}\left(p\left(K_{\Gamma / Y}+D\right)+L+c E^{\prime \prime}\right)$ is weakly positively curved on $Y$.

Proof. Let $c$ be a positive integer such that $c\left(D-E^{\prime \prime}\right)$ is Cartier. From the condition (3.3), we deduce

$$
N:=-c\left(K_{\Gamma / Y}+D\right)+c E^{\prime \prime}
$$

is a nef line bundle. We have

$$
p\left(K_{\Gamma / Y}+D\right)+L+c E^{\prime \prime}=(p+c)\left(K_{\Gamma / Y}+D\right)+(N+L) .
$$

By assumption, we can take a singular Hermitian metric $h$ on $L$ such that $\sqrt{-1} \Theta_{h}(L) \geqslant \varphi^{\star} \theta$, where $\theta$ is a smooth $(1,1)$-form on $Y$ representing $c_{1}(P)$. The multiplier ideal sheaf $\mathcal{I}\left(\left.h\right|_{\Gamma_{y}}{ }^{1 /(p+c)}\right)$ is trivial for $c$ sufficiently large (with respect to $p$ and $h$ ). Then, by applying Theorem 2.2, we conclude that $\varphi_{\star}\left((p+c)\left(K_{\Gamma / Y}+D\right)+(N+L)\right)-P$ is weakly positively curved on $Y$. 


\section{PROJECTIVE KLT PAIRS WITH NEF ANTI-CANONICAL DIVISOR}

Lemma 3.6. Let $L$ be a $\varphi$-big line bundle on $\Gamma$ and $m$ be a positive integer. Then the pushforward

$$
\pi_{\star}\left(L-\frac{1}{r} \varphi^{\star}\left(\operatorname{det} \varphi_{\star}(L+m E)\right)\right.
$$

is pseudo-effective, where $r$ is the rank of $\varphi_{\star}(L+m E)$.

Proof. Let $E_{1} \subset \Gamma$ be the union of the nonreduced locus and the nonflat locus of $\varphi: \Gamma \rightarrow Y$ (that is, the set of points $y \in Y$ at which $\varphi$ is not flat or the fiber is nonreduced). Let $\Gamma^{(r)}$ be a resolution of singularities of the $r$-times $\varphi$-fiberwise product $\Gamma \times_{Y} \cdots \times_{Y} \Gamma$, equipped with the $i$ th directional projection $\operatorname{pr}_{i}: \Gamma^{(r)} \rightarrow \Gamma$ and the natural morphism $\varphi_{r}: \Gamma^{(r)} \rightarrow Y$. Set

$$
L^{(r)}:=\sum_{i=1}^{r} \operatorname{pr}_{i}^{\star} L \quad \text { and } \quad E^{(r)}:=\sum_{i=1}^{r} \operatorname{pr}_{i}^{\star} E .
$$

For an effective divisor $E_{2}$ supported in $\sum_{i=1}^{r} \operatorname{pr}_{i}^{\star}\left(E_{1}\right)$, we define the line bundle $L^{\prime}$ by

$$
L^{\prime}:=L^{(r)}+m E^{(r)}+E_{2}-\varphi_{r}^{\star} \operatorname{det}\left(\varphi_{\star}\left(\mathcal{O}_{\Gamma}(L+m E)\right)\right) .
$$

The section $\tau \in H^{0}\left(\Gamma^{(r)}, L^{\prime}\right)$ is obtained from the natural morphism

$$
\operatorname{det} \varphi_{\star}\left(\mathcal{O}_{\Gamma}(L+m E)\right) \longrightarrow\left(\varphi_{r}\right)_{\star}\left(\mathcal{O}_{\Gamma^{(r)}}\left(L^{(r)}+m E^{(r)}+E_{2}\right)\right) .
$$

The restriction of (3.7) to a general point $y \in Y$ is nonzero, and thus $\tau$ is a nontrivial section. Hence $L^{\prime}$ is an effective line bundle.

By the assumption (3.2), there exists a (not necessarily effective) divisor $E_{3}$ supported in $\sum_{i=1}^{r} \operatorname{pr}_{i}^{\star}\left(E_{1}+E\right)$ such that

$$
-K_{\Gamma^{(r)} / Y}-\Delta^{(r)}+E_{3} \text { is nef, } \quad \text { where } \Delta^{(r)}:=\sum_{i=1}^{r} \operatorname{pr}_{i}^{\star} D .
$$

Using [Cao19, Proposition 2.10], we can take an ample line bundle $A_{Y}$ on $Y$ such that the restriction morphism

$$
\begin{array}{r}
H^{0}\left(\Gamma^{(r)}, p q\left(K_{\Gamma^{(r)} / Y}+\Delta^{(r)}\right)+p q\left(-K_{\Gamma^{(r)} / Y}-\Delta^{(r)}+E_{3}\right)+p L^{\prime}+\varphi_{r}^{\star} A_{Y}\right) \\
\longrightarrow H^{0}\left(\Gamma_{y}^{(r)}, p q E_{3}+p L^{\prime}+\varphi_{r}^{\star} A_{Y}\right)
\end{array}
$$

is surjective for any $p \in \mathbb{Z}_{+}$and for $q$ sufficiently large (with respect to $p$ ), where $\Gamma_{y}^{(r)}$ is a general fiber of $\varphi_{r}: \Gamma^{(r)} \rightarrow Y$. We consider the restriction of the above morphism to the diagonal of $\Gamma^{(r)}$ (which is well defined only on $\Gamma \backslash E_{1}$ but can be extended to $\Gamma$ by adding a divisor supported in $E_{1}$ ). Then, we choose an effective divisor $F_{p, q}$ (depending on $p$ and $q$ ) supported in $E \cup E_{1}$ such that

$$
\begin{aligned}
& H^{0}\left(\Gamma, p r L-p \varphi^{\star} \operatorname{det}\left(\varphi_{\star}\left(\mathcal{O}_{\Gamma}(L+m E)\right)\right)+\varphi^{\star} A_{Y}+F_{p, q}\right) \\
& \quad \longrightarrow H^{0}\left(\Gamma_{y}, \operatorname{pr} L-p \varphi^{\star} \operatorname{det}\left(\varphi_{\star}\left(\mathcal{O}_{\Gamma}(L+m E)\right)\right)+\varphi^{\star} A_{Y}+F_{p, q}\right)=H^{0}\left(\Gamma_{y}, p r L+F_{p, q}\right)
\end{aligned}
$$

is surjective. The right-hand side is nontrivial because $F_{p, q}$ is effective and $L$ is $\varphi$-effective. Hence, by surjectivity,

$$
\operatorname{pr} L-p \varphi^{\star} \operatorname{det}\left(\varphi_{\star}\left(\mathcal{O}_{\Gamma}(L+m E)\right)\right)+\varphi^{\star} A_{Y}+F_{p, q}
$$

is an effective line bundle. The divisor $F_{p, q}$ is contained in $E \cup E_{1}$, and thus $\pi\left(F_{p, q}\right)$ has codi- 


\section{F. Campana, J. Cao and S. Matsumura}

mension at least 2 by Theorem 3.4. Therefore, its pushforward

$$
\begin{gathered}
\pi_{*}\left(r L-\varphi^{\star} \operatorname{det}\left(\varphi_{\star}\left(\mathcal{O}_{\Gamma}(L+m E)\right)\right)+\frac{1}{p} \varphi^{\star} A_{Y}+\frac{1}{p} F_{p, q}\right) \\
=\pi_{*}\left(r L-\varphi^{\star} \operatorname{det}\left(\varphi_{\star}\left(\mathcal{O}_{\Gamma}(L+m E)\right)\right)+\frac{1}{p} \varphi^{\star} A_{Y}\right)
\end{gathered}
$$

is $\mathbb{Q}$-effective on $X$. The lemma is proved by letting $p \rightarrow+\infty$.

The following proposition, which is also a key result, follows from Lemmas 3.5 and 3.6.

Proposition 3.7. Let $A$ be the ample line bundle on $\Gamma$ chosen in Setting 3.1. There exist integers $m_{0}, m_{p} \in \mathbb{Z}_{+}$satisfying the following:

(1) The rank of $\varphi_{\star}(p A+m E)$ is independent of $m$ when $m \geqslant p m_{0}$.

(2) The natural morphism

$$
\operatorname{det} \varphi_{\star}(p A+m E) \rightarrow \operatorname{det} \varphi_{\star}(p A+(m+1) E)
$$

is isomorphic over $Y_{0}$ when $m \geqslant m_{p}$. In particular, we have

$$
\pi_{\star} \varphi^{\star} \operatorname{det}\left(\varphi_{\star}(p A+m E)\right)=\pi_{\star} \varphi^{\star} \operatorname{det}\left(\varphi_{\star}(p A+(m+1) E)\right) .
$$

(3) Fix $m_{p}$ with point (2). We define the $\mathbb{Q}$-divisor $\tilde{A}$ on $\Gamma$ by

$$
\tilde{A}:=A+m_{p} E-\frac{1}{r_{m_{0}}} \varphi^{\star} \operatorname{det} \varphi_{\star}\left(A+m_{p} E\right),
$$

where $r_{m_{0}}:=\operatorname{rank} \varphi_{\star}\left(A+m_{p} E\right)=\operatorname{rank} \varphi_{\star}\left(A+m_{0} E\right)$. Then we have

$$
\pi_{\star} \varphi^{\star} c_{1}\left(\varphi_{\star}(p \tilde{A}+\tilde{E})\right)=0
$$

for any effective $\pi$-exceptional divisor $\tilde{E}$.

Remark 3.8. Note that $p \tilde{A}$ is a Cartier divisor for $p$ divisible. Even if $p$ is not divisible, the direct image sheaf is well defined as a true direct image sheaf tensoring with a $\mathbb{Q}$-line bundle:

$$
\varphi_{\star}(p \tilde{A}+\tilde{E}):=\varphi_{\star}\left(p A+m_{0} E+\tilde{E}\right) \otimes \frac{p}{r_{m_{0}}}\left(\operatorname{det} \varphi_{\star}\left(A+m_{0} E\right)\right)^{\star} .
$$

Hence the determinant bundle and the first Chern class can also be defined.

Proof of Proposition 3.7. We first prove point (1). Since $X$ is $\mathbb{Q}$-factorial, the ample line bundle $A$ can be written as $A=\pi^{\star} B-G$ for some ample line bundle $B$ on $X$ and some effective $\pi$ exceptional divisor $G$. Let $m_{0}$ be an integer such that $m_{0} E-G$ is effective. Then $m E-p G$ is effective for any $m \geqslant p m_{0}$, as follows from $m E-p G \geqslant p\left(m_{0} E-G\right) \geqslant 0$. For a general point $y \in Y$, the restriction $\pi: \Gamma_{y} \rightarrow X_{y}$ is a birational morphism and its exceptional divisor is $\left.E\right|_{\Gamma_{y}}$. This implies that

$$
H^{0}\left(\Gamma_{y}, p A+m E\right)=H^{0}\left(\Gamma_{y}, \pi^{\star}(p B)+m E-p G\right) \cong H^{0}\left(\Gamma_{y}, \pi^{\star}(p B)\right)
$$

for any $m \geqslant p m_{0}$. Hence the rank is constant.

We now prove point (2) by contradiction. In the proof, we consider only $m$ with $m \geqslant p m_{0}$. The rank of $\varphi_{\star}(p A+m E)$ is constant, and thus the morphism (3.8) is well defined. Further, the line bundle

$$
\operatorname{det} \varphi_{\star}(p A+(m+1) E)-\operatorname{det} \varphi_{\star}(p A+m E)
$$




\section{ProjeCtive KLT PAIRS WITH NEF ANTI-CANONICAL DIVISOR}

is effective. If the morphism (3.8) is not an isomorphism over $Y_{0}$ for infinitely many $m \in \mathbb{Z}_{+}$, we can find a sequence $\left\{a_{k}\right\}_{k=1}^{\infty}$ and an effective divisor $D_{k}$ with $D_{k} \cap Y_{0} \neq \emptyset$ such that

$$
\operatorname{det} \varphi_{\star}\left(p A+\left(a_{k}+1\right) E\right) \geqslant \operatorname{det} \varphi_{\star}\left(p A+a_{k} E\right)+\left[D_{k}\right] .
$$

On the other hand, by applying Lemma 3.6 to $L:=p A$, we obtain

$$
\pi_{\star}(p A) \geqslant \frac{1}{r_{p}} \pi_{\star} \varphi^{\star} \operatorname{det} \varphi_{\star}(p A+m E),
$$

where $r_{p}$ is the rank of $\varphi_{\star}(p A+m E)$. These yield

$$
r_{p} \pi_{\star}(p A) \geqslant \pi_{\star} \varphi^{\star} \operatorname{det} \varphi_{\star}\left(p A+p m_{0} E\right)+\sum_{k=1}^{\infty} \pi_{\star} \varphi^{\star}\left[D_{k}\right] .
$$

The pushforward $\pi_{\star} \varphi^{\star}\left[D_{k}\right]$ is an effective nonzero divisor by the relation $D_{k} \cap Y_{0} \neq \emptyset$ (see the definition of $\left.Y_{0}\right)$. This gives a contradiction.

We finally prove point (3). By the construction of $\tilde{A}$, we have $\operatorname{det} \varphi_{\star}(\tilde{A})=0$. From point $(2)$, we deduce that

$$
\operatorname{det} \varphi_{\star}\left(A+m_{p} E+\tilde{E}\right) \rightarrow \operatorname{det} \varphi_{\star}\left(A+m_{p} E\right)
$$

is an isomorphism on $Y_{0}$. Here we have used that $m_{p} \geqslant m_{0}$. This implies that

$$
\pi_{\star} \varphi^{\star} \operatorname{det}\left(\varphi_{\star}(\tilde{A}+\tilde{E})\right)=\pi_{\star} \varphi^{\star} \operatorname{det} \varphi_{\star}(\tilde{A})=0 .
$$

We now show that $\pi_{\star} \varphi^{\star} \operatorname{det} \varphi_{\star}(p \tilde{A}+\tilde{E})$ is pseudo-effective. By applying Lemma 3.6 to the $\varphi$-big line bundle $A+m_{p} E$, we can find an effective $\pi$-exceptional divisor $F$ such that $\tilde{A}+F$ is pseudo-effective. By applying Lemma 3.5 to $p(\tilde{A}+F)+\tilde{E}$, we see that

$$
\varphi_{\star}\left(p(\tilde{A}+F)+\tilde{E}+c_{p} E^{\prime \prime}\right)
$$

is weakly positively curved for $c_{p}$ sufficiently large. Therefore, its determinant bundle is pseudoeffective. Using point (2), we obtain

$$
\pi_{\star} \varphi^{\star} \operatorname{det} \varphi_{\star}\left(p \tilde{A}+p F+\tilde{E}+c_{p} E^{\prime \prime}\right)=\pi_{\star} \varphi^{\star} \operatorname{det} \varphi_{\star}(p \tilde{A}+\tilde{E})
$$

since $p F+c_{p} E^{\prime \prime}$ is $\pi$-exceptional. We conclude that $\pi_{\star} \varphi^{\star} \operatorname{det} \varphi_{\star}(p \tilde{A}+\tilde{E})$ is pseudo-effective.

We finally prove that $-\pi_{\star} \varphi^{\star} \operatorname{det}\left(\varphi_{\star}(p \tilde{A}+\tilde{E})\right)$ is pseudo-effective. Applying Lemma 3.6 to the $\varphi$-big line bundle $p \tilde{A}+\tilde{E}$, we can find an effective $\pi$-exceptional divisor $F^{\prime}$ such that

$$
p \tilde{A}+\tilde{E}+F^{\prime}-\frac{1}{r_{p}} \varphi^{\star} \operatorname{det} \varphi_{\star}(p \tilde{A}+\tilde{E})
$$

is pseudo-effective, where $r_{p}$ is the rank of $\varphi_{\star}(p \tilde{A}+\tilde{E})$. We may assume that $(1 / p)\left(\tilde{E}+F^{\prime}\right)$ is a Cartier divisor on $\Gamma$ by choosing the coefficients of $F^{\prime}$ to be large and divisible. Applying Lemma 3.6 to

$$
L:=\tilde{A}+\frac{1}{p}\left(\tilde{E}+F^{\prime}\right) \quad \text { and } \quad P:=\frac{1}{p r_{p}} \operatorname{det} \varphi_{\star}(p \tilde{A}+\tilde{E})
$$

we see that

$$
\varphi_{\star}\left(\tilde{A}+\frac{1}{p}\left(\tilde{E}+F^{\prime}\right)-\frac{1}{p r_{p}} \varphi^{\star} \operatorname{det} \varphi_{\star}(p \tilde{A}+\tilde{E})\right)
$$

is pseudo-effective. By taking $\pi_{\star} \varphi^{\star} c_{1}(\bullet)$, together with (3.9), we obtain

$$
0=\pi_{\star} \varphi^{\star} c_{1}\left(\varphi_{\star}\left(\tilde{A}+\frac{1}{p}\left(\tilde{E}+F^{\prime}\right)\right)\right) \geqslant \frac{r_{m_{0}}}{p r_{p}} \pi_{\star} \varphi^{\star} c_{1}\left(\varphi_{\star}(p \tilde{A}+\tilde{E})\right),
$$




\section{F. Campana, J. Cao and S. Matsumura}

where the above inequality means that the difference is pseudo-effective. This completes the proof.

The following proposition, which is obtained from the above results, is the main result of this section.

Proposition 3.9. Let $\tilde{A}$ be the $\mathbb{Q}$-divisor defined in Proposition 3.7. Then, there exists an effective $\pi$-exceptional divisor $F$ satisfying the following: For any divisible $m \in \mathbb{Z}$ and $p \in \mathbb{Z}_{+}$, for sufficiently large $c_{m, p}>0$, the direct image sheaf

$$
\mathcal{V}_{m, p}:=\varphi_{\star}\left(-m\left(K_{\Gamma / Y}+D\right)+c_{m, p} E^{\prime \prime}+p(\tilde{A}+F)\right)
$$

is weakly positively curved and satisfies $\pi_{\star} \varphi^{\star} c_{1}\left(\mathcal{V}_{m, p}\right)=0$.

Proof. By Lemma 3.6, there exists an effective $\pi$-exceptional divisor $F$ such that $\tilde{A}+F$ is pseudoeffective (and it is also $\varphi$-big), and so Lemma 3.5 implies that $\mathcal{V}_{m, p}$ is weakly positively curved for $c_{m, p}$ sufficiently large. In particular, its first Chern class $c_{1}\left(\mathcal{V}_{m, p}\right)$ is pseudo-effective on $Y$. Hence, it is sufficient for the proof to show that $-\pi_{\star} \varphi^{\star} c_{1}\left(\mathcal{V}_{m, p}\right)$ is pseudo-effective.

Using Lemma 3.6 again, we can take an effective $\pi$-exceptional divisor $G$ such that

$$
L:=-m\left(K_{\Gamma / Y}+D\right)+c_{m, p} E^{\prime \prime}+p(\tilde{A}+F)+G-\frac{1}{r_{m, p}} \cdot \varphi^{\star} \operatorname{det} \mathcal{V}_{m, p}
$$

is pseudo-effective, where $r_{m, p}$ is the rank of $\mathcal{V}_{m, p}$. Applying Lemma 3.5 to $L$, we see that

$$
\operatorname{det}\left(\varphi_{\star}\left(m\left(K_{\Gamma / Y}+D\right)+d_{m, p} E^{\prime \prime}+L\right)\right)
$$

is pseudo-effective for $d_{m, p}$ sufficiently large. This is equivalent to saying that

$$
\operatorname{det}\left(\varphi_{\star}\left(\left(c_{m, p}+d_{m, p}\right) E^{\prime \prime}+p(\tilde{A}+F)+G\right)\right)-\frac{r_{p}}{r_{m, p}} \operatorname{det}\left(\mathcal{V}_{m, p}\right)
$$

is pseudo-effective on $Y$. From point (3) in Proposition 3.7, it follows that

$$
\pi_{\star} \varphi^{\star} c_{1}\left(\varphi_{\star}\left(\left(c_{m, p}+d_{m, p}\right) E^{\prime \prime}+p(\tilde{A}+F)+G\right)\right)=0 .
$$

Therefore, $-\pi_{\star} \varphi^{\star} c_{1}\left(\mathcal{V}_{m, p}\right)$ is pseudo-effective on $X$. This completes the proof.

\section{Structure of rationally connected fibrations}

\subsection{Proof of Theorem 1.2}

We first prove the inequality $\operatorname{nd}\left(-\left(K_{X}+\Delta\right)\right) \geqslant \operatorname{nd}\left(-\left(K_{X_{y}}+\Delta_{X_{y}}\right)\right)$ by proving the following extension theorem from a general fiber $X_{y}$ to the ambient space $X$.

Proposition 4.1. Let $(X, \Delta)$ be a projective klt pair such that $-\left(K_{X}+\Delta\right)$ is nef. Let $\psi: X \rightarrow Y$ be an almost holomorphic map to a smooth projective variety $Y$. Then, there exists an ample line bundle $B$ on $X$ such that the restriction map to a general fiber $X_{y}$ of $\psi$, that is,

$$
H^{0}\left(X,-m\left(K_{X}+\Delta\right)+B\right) \rightarrow H^{0}\left(X_{y},-m\left(K_{X_{y}}+\Delta_{X_{y}}\right)+\left.B\right|_{X_{y}}\right),
$$

is surjective for any $m \geqslant 1$ with $m\left(K_{X}+\Delta\right)$ Cartier.

In particular, we have

$$
\operatorname{nd}\left(-\left(K_{X}+\Delta\right)\right) \geqslant \operatorname{nd}\left(-\left(K_{X_{y}}+\Delta_{X_{y}}\right)\right)
$$

for a general fiber $X_{y}$ of an MRC fibration $\psi: X \rightarrow Y$ of $X$. 


\section{ProjeCtive KLT PAIRS WITH NEF ANTI-CANONICAL DIVISOR}

Remark 4.2. Proposition 4.1 can be proved for (not necessarily MRC) almost holomorphic maps. However, the proof of Theorem 1.3 requires the assumption that $\psi: X \rightarrow Y$ is an MRC fibration. The equality on the numerical dimension was recently shown for an algebraic fiber space $X \rightarrow Y$ with the nef relative anti-canonical divisor $-K_{X / Y}$ (see [EIM20]).

Proof of Proposition 4.1. Throughout the proof, let $L$ be the $\mathbb{Q}$-Cartier divisor defined by $L:=$ $-\left(K_{X}+\Delta\right)$ and $m$ be a positive integer such that $m L=-m\left(K_{X}+\Delta\right)$ is a Cartier divisor. We take a $\log$ resolution $\pi: \Gamma \rightarrow X$ of $(X, \Delta)$ that induces a resolution $\varphi: \Gamma \rightarrow Y$ of the indeterminacy locus $Z$ of $\psi$, as in the commutative diagram (3.1). Note that a general fiber $X_{y}$ of $\psi$ is compact because $\psi$ is an almost holomorphic map. For a general fiber $X_{y}$ of $\psi$, the inverse image $\Gamma_{y}:=\pi^{-1}\left(X_{y}\right)$ is also a general fiber of $\varphi$, and thus $\Gamma_{y}$ is a smooth subvariety in $\Gamma$. Further, a general fiber $\Gamma_{y}$ is not contained in the exceptional divisor $E$ of $\pi$. Then, we obtain the commutative diagram

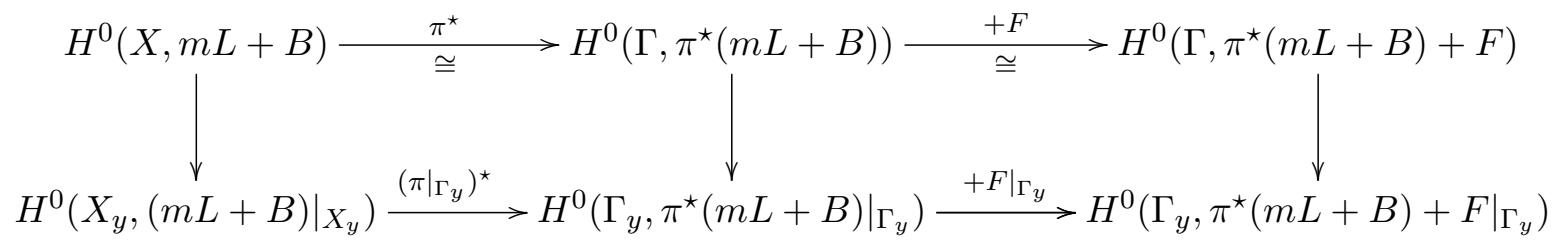

for any ample line bundle $B$ and any effective $\pi$-exceptional divisor $F$ on $\Gamma$. The horizontal maps on the bottom line are injective, and thus it is sufficient for the proof to find an ample line bundle $B$ on $X$ and an effective $\pi$-exceptional divisor $F$ on $\Gamma$ such that the rightmost vertical map is surjective.

We have the formula of canonical divisors

$$
K_{\Gamma}+\Delta^{\prime}=\pi^{\star}\left(K_{X}+\Delta\right)+E^{\prime}
$$

where $\Delta^{\prime}$ and $E^{\prime}$ are the effective divisors with no common components. The support of $\Delta^{\prime}+E^{\prime}$ is normal crossing because $\pi$ is a log resolution of $(X, \Delta)$, and we also have $\left\lfloor\Delta^{\prime}\right\rfloor=0$ because $(X, \Delta)$ has at most klt singularities. Then, for the effective $\pi$-exceptional divisor $F:=\left\lceil E^{\prime}\right\rceil$, we can easily check that

$$
F^{\prime}:=F-E^{\prime} \text { is an effective } \mathbb{Q} \text {-divisor and }\left\lfloor\Delta^{\prime}+F^{\prime}\right\rfloor=0 .
$$

In contrast, from the above formula, we have

$$
\begin{aligned}
\pi^{\star}(m L+2 B)+F & =K_{\Gamma}+\pi^{\star}(m L+2 B)+F-K_{\Gamma} \\
& =K_{\Gamma}+\pi^{\star}((m+1) L+B)+\left(\pi^{\star} B+\Delta^{\prime}+F^{\prime}\right) .
\end{aligned}
$$

We now construct a quasi-psh function $\bar{\rho}$ on $\Gamma$ and a smooth Hermitian metric $h$ on $\pi^{\star} B$ with the following properties:

- We have $\sqrt{-1} \Theta_{h}\left(\pi^{\star} B\right)+(1+\delta) d d^{c} \bar{\rho} \geqslant 0$ for any $0<\delta \ll 1$.

- The function $\bar{\rho}$ can be written as $m \log \left(\sum_{i=1}^{m}\left|z_{i}\right|^{2}\right)$ modulo the addition of smooth functions on a neighborhood of a point in $\Gamma_{y}$, where $\left(z_{1}, z_{2}, \ldots, z_{n}\right)$ are the local coordinates of $\Gamma$ such that $\Gamma_{y}=\left\{z_{1}=z_{2}=\cdots=z_{m}=0\right\}$. Here, $n:=\operatorname{dim} X$ and $m:=\operatorname{dim} Y$.

If $h$ and $\bar{\rho}$ can be constructed with the above properties, then the singular Hermitian metric

$$
H:=g_{m} h h_{\Delta^{\prime}+F^{\prime}} \text { on the line bundle } \pi^{\star}((m+1) L+B)+\left(\pi^{\star} B+\Delta^{\prime}+F^{\prime}\right)
$$

satisfies

$$
\sqrt{-1} \Theta_{H}\left(\pi^{\star}((m+1) L+B)+\left(\pi^{\star} B+\Delta^{\prime}+F^{\prime}\right)\right)+(1+\delta) d d^{c} \bar{\rho} \geqslant 0
$$




\section{F. Campana, J. Cao and S. Matsumura}

for any $0<\delta \ll 1$. Here, $g_{m}$ is a smooth Hermitian metric (obtained from the nefness of $L$ ) on the line bundle $\pi^{\star}((m+1) L+B)$ with semi-positive curvature and $h_{\Delta^{\prime}+F^{\prime}}$ is the singular Hermitian metric induced by the effective divisor $\Delta^{\prime}+F^{\prime}$. Then, by the extension theorem in [CDM17], the induced morphism

$$
\begin{aligned}
& H^{0}\left(\Gamma, \mathcal{O}_{\Gamma}\left(\pi^{\star}(m L+2 B)+F\right) \otimes \mathcal{I}(H)\right) \\
& \quad \longrightarrow H^{0}\left(\Gamma, \mathcal{O}_{\Gamma}\left(\pi^{\star}(m L+2 B)+F\right) \otimes \mathcal{I}(H) / \mathcal{I}\left(H e^{-\bar{\rho}}\right)\right)
\end{aligned}
$$

is surjective. Further, from the condition on the singularities of $\bar{\rho}$, we see that

$$
\mathcal{I}(H)=\mathcal{O}_{\Gamma} \quad \text { and } \quad \mathcal{I}\left(H e^{-\bar{\rho}}\right)=\mathcal{I}_{\Gamma_{y}} \quad \text { on a neighborhood of } \Gamma_{y}
$$

because $\Delta^{\prime}+F^{\prime}$ is a normal crossing $\mathbb{Q}$-divisor with $\left\lfloor\Delta^{\prime}+F^{\prime}\right\rfloor=0$ and a general fiber $\Gamma_{y}$ transversely intersects $\Delta^{\prime}+F^{\prime}$. This leads to the desired conclusion.

For the construction of $h$ and $\bar{\rho}$, we consider a very ample line bundle $A_{Y}$ on $Y$ and sections $\left\{s_{i}\right\}_{i=1}^{m}$ of $A_{Y}$ such that the common zero locus of $\left\{s_{i}\right\}_{i=1}^{m}$ is the disjoint union of $N$ points $\left\{y_{i}\right\}_{i=1}^{N}$ whose fibers do not intersect the indeterminacy locus $Z$ of $\psi$. Here, $N$ is the self-intersection number of $A_{Y}$. The disjoint union of the fibers $F_{i}:=\psi^{-1}\left(y_{i}\right)$ is contained in the zero locus of the section $t_{i}:=\psi^{\star} s_{i}$ of the reflexive sheaf $\left(\pi_{\star} \varphi^{\star} A_{Y}\right)^{\star \star}$. For a smooth Hermitian metric $g$, the quasi-psh function $\rho$ on $X$ defined by

$$
\rho:=m \log \sum_{i=1}^{m}\left|t_{i}\right|_{g}^{2}
$$

satisfies $(1+\delta) d d^{c} \rho \geqslant-C \sqrt{-1} \Theta_{g}$ for some constant $C>0$. Therefore, we may assume that $B$ admits a smooth Hermitian metric $g_{B}$ such that

$$
\sqrt{-1} \Theta_{g_{B}}(B)+(1+\delta) d d^{c} \rho \geqslant 0
$$

by replacing $B$ with a positive multiple of $B$ (if necessary), because $B$ is an ample line bundle on $X$. Strictly speaking, we need to deal with Hermitian metrics on complex spaces because $X$ may not be smooth. However, it is not difficult to check that the above argument still works when $X$ has singularities. Indeed, the line bundle $B$ determines the embedding of $X$ into the projective space $\mathbb{P}(|B|)$. In this case, the quasi-psh function $\rho$ and the metric $g_{B}$ can be obtained from the restriction to $X$ of $\rho$ and $g_{B}$ constructed on $\mathbb{P}(|B|)$. Then, it is easy to see that $h:=\pi^{\star} g_{B}$ and $\bar{\rho}:=\pi^{\star} \rho$ satisfy the desired properties.

In the rest of this subsection, we prove the converse inequality

$$
\operatorname{nd}\left(-\left(K_{X}+\Delta\right)\right) \leqslant \operatorname{nd}\left(-\left(K_{X_{y}}+\Delta_{X_{y}}\right)\right)
$$

by using Propositions 2.8 and 3.9. We first consider the case where $X$ is $\mathbb{Q}$-factorial. In this case, the assumptions in Setting 3.1 are automatically satisfied by setting $D:=\Delta^{\prime}$ and $E^{\prime \prime}:=E^{\prime}-\varphi^{\star} N$ (see Remark 3.3). Recall that $N$ is an effective $\mathbb{Q}$-divisor with $N \sim_{\mathbb{Q}} K_{Y}$ and that $\varphi^{\star} N$ and $E^{\prime}$ are contained in the $\pi$-exceptional divisor $E$. Let us consider the sheaf $\mathcal{W}_{m, p}$ defined by

$$
\begin{array}{r}
\mathcal{W}_{m, p}:=\varphi_{\star}\left(-m \pi^{\star}\left(K_{X}+\Delta\right)+\left(c_{m, p}-m\right) E^{\prime}+p\left(A+m_{p} E+F\right)\right. \\
\left.-\frac{p}{r_{m_{0}}} \varphi^{\star} \operatorname{det} \varphi_{\star}\left(A+m_{p} E\right)\right)
\end{array}
$$

and the reflexive hull $\left(\varphi^{\star} \mathcal{W}_{m, p}\right)^{\star \star}$.

We show that $\left(\varphi^{\star} \mathcal{W}_{m, p}\right)^{\star \star}$ satisfies the assumptions of Proposition 2.8. Let $C$ be a general curve constructed by a complete intersection, as in Proposition 2.8. By construction, the curve $C$ 


\section{ProjeCtive KLT PAIRS With NEF ANTI-CANONICAL DIVISOR}

does not intersect $E$. In particular, the curve $C$ is contained in $\Gamma_{0}:=\varphi^{-1}\left(Y_{0}\right)$ and does not intersect $\varphi^{\star} N$. We can easily check that

$$
\begin{aligned}
\mathcal{V}_{m, p}= & \varphi_{\star}\left(-m\left(K_{\Gamma / Y}+\Delta^{\prime}\right)+c_{m, p}\left(E^{\prime}-\varphi^{\star} N\right)+p(\tilde{A}+F)\right) \\
= & \varphi_{\star}\left(-m \pi^{\star}\left(K_{X}+\Delta\right)+\left(m-c_{m, p}\right) \varphi^{\star} N+\left(c_{m, p}-m\right) E^{\prime}\right. \\
& \left.\quad+p\left(A+m_{p} E+F\right)-\frac{p}{r_{m_{0}}} \varphi^{\star} \operatorname{det} \varphi_{\star}\left(A+m_{p} E\right)\right) \\
= & \mathcal{W}_{m, p} \otimes \mathcal{O}_{Y}\left(\left(m-c_{m, p}\right) N\right)
\end{aligned}
$$

by the formula (3.5) and the definitions of $\mathcal{V}_{m, p}$ and $\tilde{A}$ (see Propositions 3.7 and 3.9). Note that $\varphi^{\star} \mathcal{V}_{m, p}$ and $\varphi^{\star} \mathcal{W}_{m, p}$ are reflexive on $\Gamma_{0}$ since $\varphi: \Gamma \rightarrow Y$ is flat over $Y_{0}$. By the equality $\pi_{\star} \varphi^{\star} c_{1}\left(\mathcal{V}_{m, p}\right)=0$, we obtain $c_{1}\left(\left.\varphi^{\star} \mathcal{V}_{m, p}\right|_{C}\right)=0$. Here we have used that $C$ is contained in $\Gamma_{0}$. This implies that $c_{1}\left(\left.\varphi^{\star} \mathcal{W}_{m, p}\right|_{C}\right)=0$ since $C$ does not intersect $\varphi^{\star} N$. On the other hand, the sheaf $\mathcal{V}_{m, p}$ is weakly positively curved, hence so is $\mathcal{W}_{m, p}$ because $\left(c_{m, p}-m\right) N$ is effective by the inequality $c_{m, p} \geqslant m$. Hence, since $C$ a general curve, the restriction $\left.\varphi^{\star} \mathcal{W}_{m, p}\right|_{C}$ is also weakly positively curved. Therefore, we can conclude that $\left.\left(\varphi^{\star} \mathcal{W}_{m, p}\right)^{\star \star}\right|_{C}$ is numerically flat by Proposition 2.6.

By Proposition 2.8, we can find a constant $C$ (independent of $m$ ) such that

$$
\begin{aligned}
h^{0}\left(Y, \mathcal{W}_{m, p} \otimes \frac{p}{r_{m_{0}}} \operatorname{det} \varphi_{\star}\left(A+m_{0} E\right)\right) & \leqslant h^{0}\left(\Gamma,\left(\varphi^{\star} \mathcal{W}_{m, p}\right)^{\star \star} \otimes \frac{p}{r_{m_{0}}} \varphi^{\star} \operatorname{det} \varphi_{\star}\left(A+m_{0} E\right)\right) \\
& \leqslant C \cdot \operatorname{rank} \mathcal{W}_{m, p} \approx O\left(m^{\operatorname{nd}\left(-\left(K_{X_{y}}+\Delta_{X_{y}}\right)\right)}\right) .
\end{aligned}
$$

On the other hand, the left-hand side can be computed using the definition of a direct image sheaf. Indeed, from the definition of the numerical dimension, we can see that

$$
\begin{aligned}
& h^{0}\left(Y, \mathcal{W}_{m, p} \otimes \frac{p}{r_{m_{0}}} \operatorname{det} \varphi_{\star}\left(A+m_{0} E\right)\right) \\
& \quad=h^{0}\left(\Gamma,-m \pi^{\star}\left(K_{X}+\Delta\right)+\left(c_{m, p}-m\right) E^{\prime}+p\left(A+m_{p} E+F\right)\right) \\
& \quad=h^{0}\left(X,-m\left(K_{X}+\Delta\right)+\pi_{\star} A\right) \\
& \quad \approx O\left(m^{\operatorname{nd}\left(-\left(K_{X}+\Delta\right)\right)}\right)
\end{aligned}
$$

for $c_{m, p} \gg m>0$. Here, we have used the fact that $N, E^{\prime}$, and $E$ are $\pi$-exceptional.

Finally, we consider the case where $X$ is not $\mathbb{Q}$-factorial. In this case, following [BCHM10], we take a $\mathbb{Q}$-factorial terminal model $f:\left(X^{\prime}, \Delta^{\prime}\right) \rightarrow(X, \Delta)$ such that $K_{X^{\prime}}+\Delta^{\prime}=f^{\star}\left(K_{X}+\Delta\right)$. Then we have

$$
\operatorname{nd}\left(-\left(K_{X}+\Delta\right)\right)=\operatorname{nd}\left(-\left(K_{X^{\prime}}+\Delta^{\prime}\right)\right) \quad \text { and } \operatorname{nd}\left(-\left(K_{X_{y}}+\Delta_{X_{y}}\right)\right)=\operatorname{nd}\left(-\left(K_{X_{y}^{\prime}}+\Delta_{X_{y}^{\prime}}\right)\right),
$$

where $X_{y}^{\prime}$ is a general fiber of the composition map $X^{\prime} \rightarrow X \rightarrow Y$. Therefore, the desired conclusion follows from the case of $\mathbb{Q}$-factorial varieties.

\subsection{MRC fibrations of klt pairs $(X, \Delta)$ with smooth $X$}

In this subsection, we prove the following result, which can be seen as a generalization of [CH19, Theorem 1.2].

Theorem 4.3. Under Setting 3.1, we further assume that $X$ is smooth and that $\psi: X \rightarrow Y$ is an MRC fibration. Then, there exist subbundles $V_{1}$ and $V_{2}$ of the tangent bundle $T_{X}$ of $X$ such that $T_{X}=V_{1} \oplus V_{2}$ and $V_{1}=\pi_{\star} T_{\Gamma / Y}$. 


\section{F. Campana, J. Cao and S. Matsumura}

In particular, by [Hör07, Corollary 2.11], we can choose an MRC fibration $\psi: X \rightarrow Y$ to be (holomorphic and) locally trivial; namely, for any small (Euclidian) open set $U \subset Y$, there is a $p: \psi^{-1}(U) \cong U \times F$ that commutes with the fibrations

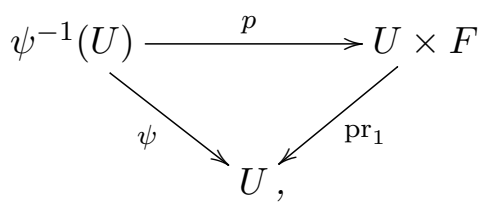

where $F$ is the fiber of $\psi$.

Remark 4.4. The main difference between Theorem 4.3 and [CH19, Theorem 1.2] is that, here, $X$ is not necessarily simply connected. The sheaves $\mathcal{V}_{m, p}$ on $Y$ in Proposition 3.9 are weakly positively curved and satisfy $\pi_{\star} \varphi^{\star} c_{1}\left(\mathcal{V}_{m, p}\right)=0$. If $X$ is simply connected, it follows that $\mathcal{V}_{m, p}$ is a trivial vector bundle over $Y_{0}$, and then $\varphi: \Gamma \rightarrow Y$ is birational to a product. In the case where $X$ is not simply connected, the argument is more involved.

Proof of Theorem 4.3. We consider the direct image sheaf $\mathcal{V}_{p}$ defined by

$$
\mathcal{V}_{p}:=\mathcal{V}_{0, p}=\varphi_{\star}\left(c_{0, p} E^{\prime \prime}+p(\tilde{A}+F)\right),
$$

where $\tilde{A}$ is the $\mathbb{Q}$-divisor defined in Proposition 3.7. The sheaf $\pi_{\star} \varphi^{\star} \mathcal{V}_{p}$ is weakly positively curved and satisfies $c_{1}\left(\pi_{\star} \varphi^{\star} \mathcal{V}_{p}\right)=0$ by Proposition 3.9. Hence, by Proposition 2.6, the reflexive hull $\left(\pi_{\star} \varphi^{\star} \mathcal{V}_{p}\right)^{\star \star}$ is a numerically flat vector bundle on $X$. In particular, it comes equipped with a holomorphic flat connection. The morphism $\varphi: \Gamma \rightarrow Y$ is flat over $Y_{0}$ (see the definition of $Y_{0}$ ), and so the pull-back $\varphi^{\star} \mathcal{V}_{p}$ is reflexive on $\Gamma_{0}:=\varphi^{-1}\left(Y_{0}\right)$. Hence we have

$$
\varphi^{\star} \mathcal{V}_{p}=\pi^{\star}\left(\pi_{\star} \varphi^{\star} \mathcal{V}_{p}\right)^{\star \star} \text { on } \Gamma_{0} \backslash E,
$$

and thus there exists a flat connection $\nabla$ of $\varphi^{\star} \mathcal{V}_{p}$ on $\Gamma_{0} \backslash E$.

Our goal is to show that the flat connection $\nabla$ induces the flatness of $\mathcal{V}_{p}$ on $Y_{0}$ except for a subvariety of codimension at least 2 . The problem is local in $Y_{0}$, and thus we assume that $Y_{0}$ is a sufficiently small open set. Further, we can assume that $\mathcal{V}_{p}$ is locally free over $Y_{0}$ after removing a subvariety of codimension at least 2 . Let $\nabla_{H}$ be the Chern connection of $\varphi^{\star} \mathcal{V}_{p}$ associated with the Hermitian metric $H:=\varphi^{\star} h$, where $h$ is a Hermitian metric on $\mathcal{V}_{p}$. Let

$$
M_{H} \in C_{1,0}^{\infty}\left(\Gamma_{0}, \operatorname{End}\left(\varphi^{\star} \mathcal{V}_{p}\right)\right) \quad \text { and } \quad M \in C_{1,0}^{\infty}\left(\Gamma_{0} \backslash E, \operatorname{End}\left(\varphi^{\star} \mathcal{V}_{p}\right)\right)
$$

be the connection forms of $\nabla_{H}$ and $\nabla$, respectively. Then, we have

$$
\nabla=\nabla_{H}+M-M_{H} \quad \text { on } \Gamma_{0} \backslash \varphi^{-1}(\varphi(E)) .
$$

It follows from $H=\varphi^{\star} h$ that $\nabla_{H}$ and $M_{H}$ can be obtained from the pull-backs.

We first show that the connection form $M$ descends to a Zariski open set in $Y_{0} \backslash \varphi(E)$. Note that $\varphi(E)$ is properly contained in $Y_{0}$ since $X$ is smooth. For this purpose, we fix a local frame of $\mathcal{V}_{p}$ and consider the frame of $\varphi^{\star} \mathcal{V}_{p}$ induced by its pull-back. Then, with respect to these trivializations of $\mathcal{V}_{p}$ and $\varphi^{\star} \mathcal{V}_{p}$, the connection form $M$ can be regarded as a matrix of the $(1,0)$-forms $M_{i j}$. The connection form $M$ satisfies

$$
d M+M \wedge M=0
$$

because $\nabla$ is a flat connection. The $(1,1)$-part of the left-hand side is $\bar{\partial} M$ because $M$ is of (1,0)-type. This implies that $M_{i j}$ is a holomorphic 1-form on $\Gamma_{0} \backslash E$. 


\section{PROJECTIVE KLT PAIRS WITH NEF ANTI-CANONICAL DIVISOR}

Let $Y_{1}$ be a nonempty Zariski open set in $Y_{0} \backslash \varphi(E)$ consisting of the points $y$ whose fiber $\Gamma_{y}$ is smooth and rationally connected. The fiber $\Gamma_{y}$ at $y \in Y_{1}$ has no holomorphic form as it is a rationally connected manifold, and thus the restriction $\left.M_{i j}\right|_{\Gamma_{y}}$ should be identically zero. This implies that there exist holomorphic 1 -forms $\eta_{i j}$ on $Y_{1}$ such that $M_{i j}=\varphi^{\star} \eta_{i j}$ holds.

The remaining problem is to extend $\eta_{i j}$ to a holomorphic 1-form on $Y_{0}$ (except for a subvariety of codimension at least 2). Let $P$ be a divisorial component of $\left(Y_{1} \backslash Y_{0}\right) \cup \varphi(E)$ on $Y_{0}$. It suffices to show that $\eta_{i j}$ can be extended through a general point in $P$. For a general point $y \in P$, we can find a point $x \in \Gamma_{y}$ such that $x \notin E$ and $\varphi: X \rightarrow Y$ is smooth at $x$, by Theorem 3.4(2). There exists a local section of $\varphi: \Gamma \rightarrow Y$ from a neighborhood of $y$ to one of $x$. By pulling back $M_{i j}=\varphi^{\star} \eta_{i j}$ with this local section, we see that $\eta_{i j}$ is bounded on a neighborhood of $y$, which can be extended by the Riemann extension theorem. Here we have used that $M_{i j}$ is defined on $\Gamma_{0} \backslash E$ (and not only on $\Gamma_{1}:=\varphi^{-1}\left(Y_{1}\right)$ ). Hence $M=\varphi^{\star}\left[\eta_{i j}\right]$ holds outside a subvariety of codimension at least 2 . For the Chern connection $\nabla_{h}$ of $\left(\left.\mathcal{V}_{p}\right|_{Y_{0}}, h\right)$, we can easily check that $\nabla_{h}+\left[\eta_{i j}\right]$ determines a flat connection of $\mathcal{V}_{p}$.

By the above argument, our situation is the same as in [CH19, Section 3.C]; namely, $\mathcal{V}_{p}=$ $\varphi_{\star}\left(c_{0, p} E^{\prime}+p(\tilde{A}+F)\right)$ is flat on $Y_{0}$. Note that the $\pi$-exceptional divisor $E$ (and thus $E^{\prime \prime}$ and $F$ ) is not dominant over $Y$ since $X$ is smooth. Following the same argument as in [CH19, Section 3.C], we see that the flat connection of $\mathcal{V}_{p}$ on $Y_{0}$ induces a birational morphism from $\varphi: \Gamma_{0} \rightarrow Y_{0}$ to a locally trivial fibration, and the birational morphism does not contract any non- $\pi$-exceptional divisors on $\Gamma_{0}$. This induces a splitting $T_{X}=V_{1} \oplus V_{2}$ on $X \backslash S$ for some subvariety $S$ of codimension at least 2. As $X$ is smooth, the splitting can be extended to the total space. This finishes the proof.

\subsection{Proof of Theorem 1.3}

Theorem 4.3 allows us to take a locally trivial MRC fibration $\psi: X \rightarrow Y$ to a smooth projective variety $Y$. Hence $\pi: \Gamma \rightarrow X$ in Setting 3.1 can be chosen as the identity map of $X$.

We first show that $c_{1}(Y)=0$ and $\Delta$ is $\psi$-horizontal. Let $\Delta=\Delta^{\text {hor }}+\Delta^{\text {vert }}$ be the decomposition of $\Delta$ into the vertical part $\Delta^{\text {vert }}$ and the horizontal part $\Delta^{\text {hor }}$. Then, we have

$$
-\Delta^{\text {vert }}-\psi^{\star} K_{Y}=K_{X / Y}-\left(K_{X}+\Delta\right)+\Delta^{\text {hor }} \text {. }
$$

The restriction of $K_{X / Y}-\left(K_{X}+\Delta\right)+\Delta^{\text {hor }}$ to a general fiber of $\psi$ is effective. Hence, by [BP08], we see that $-\Delta^{\text {vert }}-\psi^{\star} K_{Y}$ is pseudo-effective. However, as $Y$ is the base of MRC fibrations, the variety $Y$ is not uniruled by [GHS03], and thus $K_{Y}$ is pseudo-effective by [BDPP13]. As a consequence, we see that $\Delta^{\text {vert }}=0$ and $c_{1}(Y)=0$.

Finally, we prove that the identification $p: \psi^{-1}(U) \cong U \times F$ in Theorem 4.3 also identifies the boundary $\Delta$ by applying Proposition 2.7. In our situation, the $\mathbb{Q}$-divisor $\tilde{A}$ constructed in Proposition 3.7 can be written as

$$
\tilde{A}=A-\frac{1}{r_{m_{0}}} \psi^{\star} \operatorname{det} \psi_{\star} A
$$

Further, $\tilde{A}$ is pseudo-effective and $\psi_{\star}(p \tilde{A})$ is numerically flat for any $p \in \mathbb{Z}_{+}$.

Let $\Delta=\sum a_{i} \Delta_{i}$ be the irreducible decomposition. We now prove that $\psi_{\star}\left(q \Delta_{i}+p \tilde{A}\right)$ is a numerically flat vector bundle on $Y$ for any divisible $p, q \in \mathbb{Z}_{+}$and any $i$. By Lemma 3.5, the direct image $\psi_{\star}\left(q \Delta_{i}+p \tilde{A}\right)$ is weakly positively curved. It suffices to show that $c_{1}(P)=0$ by Proposition 2.6, where $P$ is the line bundle defined by $P:=\operatorname{det}\left(\psi_{\star}\left(q \Delta_{i}+p \tilde{A}\right)\right)$. Note that $P$ 


\section{F. Campana, J. Cao and S. Matsumura}

is pseudo-effective. By applying Lemma 3.6 to $q \Delta_{i}+p \tilde{A}$, we see that

$$
L:=q \Delta_{i}+p \tilde{A}-\frac{1}{r} \psi^{\star} P
$$

is pseudo-effective, where $r$ is the rank of $\psi_{\star}\left(q \Delta_{i}+p \tilde{A}\right)$. Note that $L$ is $\psi$-big by definition. Applying Theorem 2.2 to $m \gg p$ and the form

$$
p \tilde{A}-\frac{1}{r} \psi^{\star} P=m K_{X / Y}-m\left(K_{X / Y}+\Delta\right)+\left(m \Delta-q \Delta_{i}\right)+L,
$$

we see that $\psi_{\star}\left(p \tilde{A}-(1 / r) \psi^{\star} P\right)$ is weakly positively curved. This implies that

$$
c_{1}\left(\psi_{\star}(p \tilde{A})\right)-\frac{r_{p}}{r} c_{1}(P)
$$

is pseudo-effective, where $r_{q}$ is the rank of $\psi_{\star}(p \tilde{A})$. This implies that $c_{1}(P)=0$ because $c_{1}\left(\psi_{\star}(p \tilde{A})\right)=0$ by Proposition 3.7. Then, by applying Proposition 2.7, Theorem 1.3 is proved.

\section{Structure of slope rationally connected quotients}

\subsection{Orbifold surfaces with nef anti-canonical divisor}

This section is devoted to the proof of Conjecture 1.5 for projective surfaces. Theorem 1.6 can be derived from Theorems 5.1 and 5.3.

ThEOREM 5.1. Let $(S, D)$ be a $\log$ smooth klt pair such that $S$ is a surface and $-\left(K_{S}+D\right)$ is nef. Then, there exists an orbifold morphism $\rho:(S, D) \rightarrow\left(R, D_{R}\right)$ onto the orbifold base $\left(R, D_{R}\right)$ with the following properties:

(1) The pair $\left(R, D_{R}\right)$ is a klt pair such that $R$ is smooth and $c_{1}\left(K_{R}+D_{R}\right)=0$.

(2) The general orbifold fibers $\left(S_{r}, D_{r}\right)$ are $s R C$.

(3) The morphism $\rho$ is locally trivial with respect to pairs; namely, for any small (Euclidean) open set $U \subset R$, there is an isomorphism

$$
\left(\rho^{-1}(U), D\right) \cong\left(U,\left.D_{R}\right|_{U}\right) \times\left(S_{r},\left.D\right|_{S_{r}}\right)
$$

over $U \subset R$. Here $S_{r}$ is a general fiber of $\rho$.

In particular, Conjecture 1.5 is true in the 2-dimensional case.

Remark 5.2. Example 5.10 at the end of this subsection shows that the conclusion of Conjecture 1.5 fails for (non-klt) lc pairs.

Proof of Theorem 5.1. We first consider the case where $h^{1}\left(S, \mathcal{O}_{S}\right) \neq 0$. In this case, the surface $S$ is not rationally connected. Hence, by applying Theorem 1.3, we can find a locally trivial MRC fibration $\psi: S \rightarrow Y$ onto a smooth manifold $Y$ such that $c_{1}(Y)=0$ and $\operatorname{dim} Y>0$. Notice, however, that the arguments of the proof of Theorem 5.3 below also apply in a simpler way when $h^{1}\left(S, \mathcal{O}_{S}\right)>0$ and permit us to obtain the conclusion without using Theorem 1.3.

If $\operatorname{dim} Y=2$, the sRC quotient of $S$ coincides with the MRC fibration. Indeed, in this case, the surface $S$ is not uniruled, and thus the canonical divisor $K_{S}$ is pseudo-effective. Hence, we see that $-D=-\left(K_{S}+D\right)+K_{S}$ is pseudo-effective. This implies that $D=0$, and thus the identity map of $S$ is the sRC quotient. 


\section{ProjeCtive KLT PAIRS WITH NEF ANTI-CANONICAL DIVISOR}

If $\operatorname{dim} Y=1$, all the fibers $F$ of $\psi$ are copies of $\mathbb{P}^{1}$. If $-\left.\operatorname{deg}\left(K_{S}+D\right)\right|_{F}>0$, we have

$$
2-\left.\operatorname{deg} D\right|_{F}=-\operatorname{deg}\left(K_{F}+\left.D\right|_{F}\right)=-\left.\operatorname{deg}\left(K_{S}+D\right)\right|_{F}>0 .
$$

Then, all the fibers are sRC. Hence, the MRC fibration $\psi$ itself gives the sRC quotient and satisfies all the conditions of the theorem.

If $-\left.\operatorname{deg}\left(K_{S}+D\right)\right|_{F}=0$, then $-\left(K_{S}+D\right)$ is numerically equivalent to $a F$ because $\psi: S \rightarrow Y$ is locally trivial. Note that $a \geqslant 0$ since $-\left(K_{S}+D\right)$ is nef.

In the case where $a>0$, the pair $(S, D+\delta F)$ is still a klt pair with nef anti-log canonical divisor for sufficiently small $\delta>0$. By Theorem 1.3, the boundary divisor $D+\delta F$ should be horizontal with respect to the MRC fibration, which gives a contradiction. Hence we obtain $a=0$ (that is, $-\left(K_{S}+D\right)$ is numerically trivial). Then, the identity map of $S$ gives the sRC quotient and satisfies all the conditions of the theorem.

Let us consider the remaining case where $h^{1}\left(S, \mathcal{O}_{S}\right)=0$. Then we can obtain a more precise decomposition in the following form. Theorem 5.3 completes the proof of Theorem 5.1.

The remainder of this subsection is devoted to the proof of the following theorem.

THEOREM 5.3. Let $(S, D)$ be a $\log$ smooth klt pair such that $S$ is a compact Kähler surface and $-\left(K_{S}+D\right)$ is nef. We assume $h^{1}\left(S, \mathcal{O}_{S}\right)=0$ and that $(S, D)$ is not sRC. Then, the pair $(S, D)$ is a product with respect to klt pairs $\left(B, D_{B}\right)$ and $\left(\mathbb{P}^{1}, D_{\mathbb{P}^{1}}\right)$ :

$$
(S, D)=\left(B, D_{B}\right) \times\left(\mathbb{P}^{1}, D_{\mathbb{P}^{1}}\right) ;
$$

that is, $S=B \times \mathbb{P}^{1}$ and $D=p^{\star} D_{B}+q^{\star} D_{\mathbb{P}^{1}}$.

Proof. By applying [Cam17, Theorem 1.5] (see also Theorem 1.4) to $(S, D)$, we obtain a birational orbifold morphism $g:\left(S^{\prime}, D^{\prime}\right) \rightarrow(S, D)$ with the following properties:

(1) We have $g_{\star} D^{\prime}=D$.

(2) The surface $S^{\prime}$ is smooth and $\left(S^{\prime}, D^{\prime}=\bar{D}+\Delta^{\prime}\right)$ is klt.

(3) We have $g^{\star}\left(K_{S}+D\right)=K_{S^{\prime}}+\bar{D}+\Delta^{\prime}-E^{\bullet}$.

Here $\bar{D}$ is the strict transform of $D$, and $\Delta^{\prime}$ and $E^{\bullet}$ are effective $g$-exceptional $\mathbb{Q}$-divisors with no common components. Moreover, we can take an orbifold morphism $f:\left(S^{\prime}, D^{\prime}\right) \rightarrow\left(B, D_{B}\right)$ satisfying the following commutative diagram:

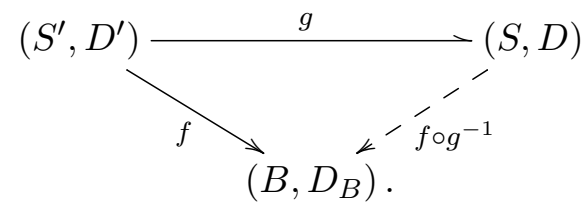

In contrast, from [Cam17, Corollary 10.4] and the assumption of nefness, we obtain

$$
c_{1}\left(K_{B}+D_{B}\right)=0 .
$$

Therefore, there are two possibilities: either $B=\mathbb{P}^{1}$ with $\operatorname{deg} D_{B}=2$, or $B$ is an elliptic curve with $D_{B}=0$. This second case is excluded by the assumption $h^{1}\left(S, \mathcal{O}_{S}\right)=0$. The proof of the theorem consists of the following steps:

Step 1. Using Lemma 5.4, we show that the rational map $f \circ g^{-1}: S \rightarrow B$ is actually holomorphic. We may then assume $g=\operatorname{id}_{S}, S^{\prime}=S$, and $D^{\prime}=D$. 


\section{F. Campana, J. Cao and S. Matsumura}

Then, $f: S \rightarrow B$, which is a (not necessarily minimal) ruled surface, can be factorized into a sequence of blow-downs $g: S \rightarrow P$ and a $\mathbb{P}^{1}$-bundle $h: P \rightarrow B$. Here $P$ is a smooth surface and $f=h \circ g$ holds (see the following diagram). We define the divisor $D_{P}$ on $P$ by $D_{P}:=g_{\star} D$.

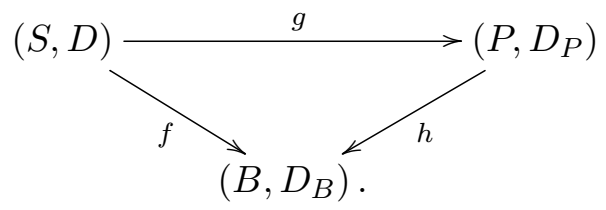

Step 2. Using Lemma 5.5, we show that $-\left(K_{P}+D_{P}\right)$ is nef.

Step 3. Using Lemma 5.7, we show that $\left(P, D_{P}\right)$ is a product of $\left(B, D_{B}\right)$ and $\left(\mathbb{P}^{1}, D_{\mathbb{P}^{1}}\right)$, as in the statement of Theorem 5.3. Here $D_{\mathbb{P}^{1}}$ is the restriction of $D_{P}$ to a general fiber of $h: P \rightarrow B$.

Step 4. Using Lemma 5.9, we show that $g=\operatorname{id}_{P}$ and $S=P$.

Let us prove the four lemmas mentioned in these steps, using the same notation as above.

LEMMA 5.4. In the same situation as above, the rational map $f \circ g^{-1}$ is a holomorphic map (without indeterminacy locus).

Proof. We assume that the indeterminacy locus of $f \circ g^{-1}$ is nonempty. Then, we can choose a $g$-exceptional component $E \cong \mathbb{P}^{1}$ of either $E^{\bullet}$ or $\Delta^{\prime}$ such that $f(E)=B$. Recall that the support of $\bar{D}+\Delta^{\prime}+E^{\bullet}$ is simple normal crossing and that the coefficients of $\bar{D}$ lie in $[0,1)$. The divisor $E$, being $g$-exceptional, meets at most two other components of $\bar{D}$, each of which has a coefficient in $[0,1)$. Hence, in the first case (that is, $\left.E \subset E^{\bullet}\right)$, see that $\operatorname{deg}\left(K_{E}+\left.\bar{D}\right|_{E}\right)<0$. In the second case (that is, $E \subset \Delta^{\prime}$ ), we set $D^{\prime \prime}:=\bar{D}-c E$, where $c \in[0,1)$ is the coefficient of $E$ in $\bar{D}$. Then, we have $\operatorname{deg}\left(K_{E}+\left.D^{\prime \prime}\right|_{E}\right)<0$ for the same reason.

Let us now consider the case where $E \subset \Delta^{\prime}$. As $B$ is a curve, there are no $f$-exceptional divisors on $S^{\prime}$. Further, $\left(B, D_{B}\right)$ is the orbifold base of $\left(S^{\prime}, D^{\prime}\right)$ and $E$ is $f$-horizontal. Hence, the natural maps $\left(S^{\prime}, D^{\prime}\right) \rightarrow\left(B, D_{B}\right)$ and $\left(S, D^{\prime \prime}\right) \rightarrow\left(B, D_{B}\right)$ are orbifold morphisms with the same orbifold base. By considering the restriction of $f$ to $E$, we obtain the orbifold morphism $m:\left(E, D^{\prime \prime}\right) \rightarrow\left(B, D_{B, E}\right)$ with the orbifold divisor $D_{B, E}$. The divisor $E$ is $f$-horizontal, and all components of $D^{\prime \prime}$ transversely intersect $E$. Further, we can take a fiber $F_{b}:=f^{-1}(b)$ of $b \in B$ that intersects $E$, and thus we have

$$
c_{F}\left(F_{b}\right) \cdot c_{D^{\prime}}\left(F_{b}\right) \geqslant c_{D_{B}}\left(F_{b}\right),
$$

where $c_{F}\left(F_{b}\right)$ (respectively, $c_{D^{\prime}}\left(F_{b}\right), c_{D_{B}}\left(F_{b}\right)$ ) is the multiplicity of $F$ in $f^{\star}(b)$ (respectively, $D^{\prime}$, $\left.D_{B}\right)$. Then, we obtain $D_{B, E} \geqslant D_{B}$ from the above inequality.

As $E$ and $B$ have the same dimension (1), we find that $m^{\star}\left(K_{B}+D_{B, E}\right) \leqslant\left(K_{E}+\left.D^{\prime \prime}\right|_{E}\right)$ and thus obtain

$$
0=\operatorname{deg}\left(m^{\star}\left(K_{B}+D_{B}\right)\right) \leqslant \operatorname{deg}\left(m^{\star}\left(K_{B}+D_{B, E}\right)\right) \leqslant \operatorname{deg}\left(K_{E}+\left.D^{\prime \prime}\right|_{E}\right)<0 .
$$

This gives a contradiction.

For the remaining case (that is, $E \subset E^{\bullet}$ ), the same argument works when applied to $K_{E}+\left.D^{\prime}\right|_{E}$ (instead of $K_{E}+\left.D^{\prime \prime}\right|_{E}$ ). This concludes the proof of the lemma.

Lemma 5.5. Let $(S, D)$ be an arbitrary orbifold pair such that $S$ is a smooth surface and $-\left(K_{S}+D\right)$ is nef. Let $g: S \rightarrow T$ be the contraction of a $(-1)$-curve of $S$. Then, $-\left(K_{T}+D_{T}\right)$, where $D_{T}:=g_{\star} D$, is nef. In particular, the divisor $-\left(K_{P}+D_{P}\right)$ is nef in the above situation. 


\section{PROJECTIVE KLT PAIRS WITH NEF ANTI-CANONICAL DIVISOR}

Proof. By assumption, we have $K_{S}+D=g^{\star}\left(K_{T}+D_{T}\right)+\ell E$ for some $\ell \in \mathbb{Q}$. Then, $\ell$ is nonnegative since $0 \leqslant-\left(K_{S}+D\right) \cdot E=-\ell \cdot E^{2}=\ell$. Therefore, we see that $-g^{\star}\left(K_{T}+D_{T}\right)=$ $-\left(K_{S}+D_{S}\right)+\ell E$ is nef by the projection formula. This leads to the lemma.

Remark 5.6. Even in the case where $h^{1}\left(S, \mathcal{O}_{S}\right) \neq 0$, Lemma 5.4 is true, and its proof is much easier. Further, Lemmas 5.4 and 5.5 are true in more general situations: Lemma 5.4 holds for any klt surface pair, and Lemma 5.5 holds for any $K$-negative contractions by the "negativity lemma."

Lemma 5.7. Let $h: P \rightarrow B$ be a $\mathbb{P}^{1}$-bundle over a smooth curve $B=\mathbb{P}^{1}$. Let $D_{P}$ be a (not necessarily snc-supported) klt orbifold divisor on $P$ with $-\left(K_{P}+D_{P}\right)$ nef and $D_{B}$ be the divisor on $B$ that gives the orbifold base of $\left(P, D_{P}\right)$. Then, $\left(P, D_{P}\right)$ is isomorphic to the product orbifold $\left(B, D_{B}\right) \times\left(\mathbb{P}^{1}, D_{\mathbb{P}^{1}}\right)$.

Proof. We may assume that $P$ is the Hirzebruch surface $\mathbb{F}_{m}$ containing a section $C \subset P=\mathbb{F}_{m}$ satisfying $C^{2}=-m$, where $m \geqslant 0$. The divisor $D_{P}$ can be decomposed into the vertical part $D_{P}^{\text {vert }}$ and the horizontal part $D_{P}^{\text {hor }}$. Then, we have

$$
D_{P}^{\text {vert }}=p^{\star} D_{B} \quad \text { and } \quad D_{P}^{\text {hor }}=a C+\sum_{j} a_{j} H_{j},
$$

where $a$ and the $a_{j}$ are positive rational numbers and the $H_{j}$ are distinct irreducible curves. It follows from $H_{j} \cdot C \geqslant 0$ that each $H_{j}$ is numerically equivalent to $d_{j}\left(C+b_{j} F\right)$ for some rational number $b_{j} \geqslant m$; we denote this equivalence by

$$
H_{j} \equiv d_{j}\left(C+b_{j} F\right),
$$

where $F$ is a general fiber of $h: P \rightarrow B$ and $d_{j}$ is the positive integer defined by the intersection number $d_{j}:=H_{j} \cdot F$. Then, we have

$$
-\left(K_{P}+D_{P}\right) \equiv 2 C+(m+2) F-2 F-\left(a C+\sum_{j} a_{j} d_{j}\left(C+b_{j} F\right)\right) .
$$

By computing the intersection of $-\left(K_{P}+D_{P}\right)$ with $C$, we obtain

$$
\begin{aligned}
0 \leqslant-\left(K_{P}+D_{P}\right) \cdot C & =-m+\left(a+\sum_{j} a_{j} d_{j}\right) m-\sum_{j} a_{j} b_{j} d_{j} \\
& =-m+a m+\sum_{j} a_{j} d_{j} \cdot\left(m-b_{j}\right) .
\end{aligned}
$$

This implies that $m=0$ because $b_{j} \geqslant m$ and $a<1$ (which comes from the klt condition). Therefore, we conclude that $P=\mathbb{F}_{0}=\mathbb{P}^{1} \times \mathbb{P}^{1}$. Further, we see that $b_{j}=0$, and thus $H_{j} \equiv C$ for any $j$.

Remark 5.8. In the case where $B$ is an elliptic curve, the previous computation shows that either $\left(P, D_{P}\right)$ is a product, or $P$ is the "non-decomposable" ruled surface over $B$ with minimal selfintersection curve $C$ such that $C^{2}=0$ or $C^{2}=1$. Further, if $C^{2}=0$, we see that $D_{P}=0$ by the same argument.

In our situation, the canonical divisor $\left(K_{B}+D_{B}\right)$ is numerically trivial (see (5.1)), by the argument in the proof of Theorem 5.3. Hence, $B=\mathbb{P}^{1}$ with $\operatorname{deg} D_{B}=2$, or $B$ is an elliptic curve with $D_{B}=0$. The latter case contradicts the assumption $0=h^{1}\left(S, \mathcal{O}_{S}\right)=h^{1}\left(P, \mathcal{O}_{P}\right)$. Hence, it is sufficient to consider the former case $B=\mathbb{P}^{1}$ for the proof of Theorem 5.3. 


\section{F. Campana, J. Cao and S. Matsumura}

Lemma 5.9. Let $\left(P, D_{P}\right)$ be a product orbifold $\left(B, D_{B}\right) \times\left(\mathbb{P}^{1}, D_{\mathbb{P}^{1}}\right)$, where both factors are klt orbifold curves with $\operatorname{deg}\left(K_{B}+D_{B}\right)=0$. Let $(S, D)$ be a klt pair such that $S$ is smooth and $-\left(K_{S}+D\right)$ is nef. If $g:(S, D) \rightarrow\left(P, D_{P}\right)$ is a birational orbifold morphism satisfying $D_{P}=g_{\star} D$ and $\left(B, D_{B}\right)$ is the orbifold base of $g \circ p$, then we have $S=P, g=\mathrm{id}_{P}$, and $D=D_{P}$.

Proof. The birational morphism $g$ is the composition of blow-ups of points, and thus, by Lemma 5.5, it is sufficient to prove the lemma when $g$ is the blow-up $g: S \rightarrow P=B \times \mathbb{P}^{1}$ at one point $r \in P$. We have a commutative diagram

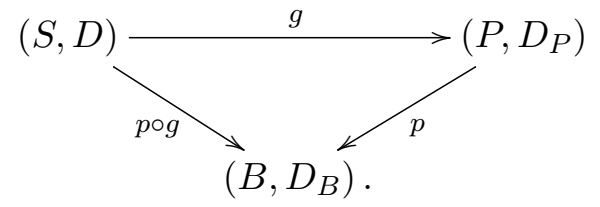

Let $E$ be the exceptional divisor of $g: S \rightarrow P=B \times \mathbb{P}^{1}$ at $r \in P$. Let $F^{\prime}$ (respectively, $C^{\prime}$ ) be the strict transform in $S$ of the fiber $F$ (respectively, $C$ ) of $p$ (respectively, $q$ ) passing through the blow-up center $r$, where $p$ (respectively, $q$ ) is the first (respectively, second) projection of the product $B \times F$. Further, let $m_{F} \in[1, \infty]$ (respectively, $m_{C} \in[1, \infty]$ ) be the multiplicity of $F$ (respectively, $C$ ) in $D_{P}$.

By assumption, the divisor $D_{P}$ can be obtained from the sum of the pull-backs of $D_{B}$ and $D_{\mathbb{P}^{1}}$, and thus we have

$$
D_{P}=\Gamma+\left(1-\frac{1}{m_{F}}\right) F+\left(1-\frac{1}{m_{C}}\right) C,
$$

where $\Gamma$ is the effective divisor whose support does not contain the point $r$. We remark that the case where $m_{F}=1$ (respectively, $m_{C}=1$ ) corresponds to the case where $r$ does not lie in the vertical divisor $D_{P}^{\text {vert }}$ (respectively, in the horizontal divisor $D_{P}^{\text {hor }}$ ). On the other hand, when we define $\delta$ as the intersection number $\delta:=D_{P}^{\text {hor }} \cdot F$, we have

$$
D_{P}=p^{\star}\left(D_{B}\right)+q^{\star}\left(D_{\mathbb{P}^{1}}\right) \equiv 2 F+\delta C
$$

by the equality $\operatorname{deg}\left(K_{B}+D_{B}\right)=0$.

We now compute the numerical class of $-\left(K_{S}+D\right)$ in $H^{1,1}(S, \mathbb{Q})$, which is spanned over $\mathbb{Q}$ by the numerical classes of $F^{\prime}, C^{\prime}$, and $E$. When we define $\bar{D}$ as the strict transform of $D_{P}$, we have $K_{S}+D=K_{S}+\bar{D}+s E$ for some $1>s \geqslant 0$. A straightforward computation yields

$$
\begin{gathered}
K_{S}=-2\left(F^{\prime}+E\right)-2\left(C^{\prime}+E\right)+E=-\left(2 F^{\prime}+2 C^{\prime}+3 E\right), \\
\bar{D} \equiv 2\left(F^{\prime}+E\right)+\delta\left(C^{\prime}+E\right)-\left(\left(1-\frac{1}{m_{F}}\right) E+\left(1-\frac{1}{m_{C}}\right) E\right)=2 F^{\prime}+\delta C^{\prime}+(\delta+\mu) E,
\end{gathered}
$$

where $\mu:=\left(1 / m_{F}+1 / m_{C}\right)$. Hence we obtain

$$
-\left(K_{S}+D\right) \equiv(2-\delta) C^{\prime}+(3-(\delta+\mu+s)) E .
$$

By using the equalities $C^{2}=E^{2}=-1$ and $C^{\prime} \cdot E=1$ of the intersection numbers, we see that

$$
-\left(K_{S}+D\right) \cdot E=\left(K_{S}+D\right) \cdot C^{\prime}=-1+(\mu+s) .
$$

Hence $1=\mu+s$ follows from the assumption that $-\left(K_{S}+D\right)$ is nef.

We denote by $t$ the multiplicity of $E$ in $D$ (that is, $1-1 / t=s$ ). Then, we have $t \geqslant m_{F}$ since $\left(B, D_{B}\right)$ is the orbifold base of $p \circ g:(S, D) \rightarrow\left(B, D_{B}\right)$. Together with the equality $s+\mu=1$, 


\section{ProjeCtive KLT PAIRS With NEF ANTI-CANONICAL DIVISOR}

this gives

$$
\frac{1}{m_{F}} \geqslant \frac{1}{t}=\frac{1}{m_{F}}+\frac{1}{m_{C}} .
$$

Hence we see that $t=m_{F}$ and $1 / m_{C}=0$, which contradicts the klt property of $(S, D)$.

Example 5.10. We now construct a smooth lc orbifold surface $(S, D)$ with nef anti-log canonical divisor, so that $S$ is rationally connected but its sRC quotient does not decompose $S$ as a product.

Starting from $S_{0}=\mathbb{P}^{1} \times \mathbb{P}^{1}$ with projections $p$ and $q$ onto its first and second factors, let $D_{0}=V+V^{\prime}+H$, where $V$ and $V^{\prime}$ are two (reduced) fibers of $p$ and $H$ is a fiber of $q$. Then, we have $K_{S_{0}}+D_{0}=-H_{0}$. Let $g: S \rightarrow S_{0}$ be the blow-up of the two intersection points of $H$ with $V$ and $V^{\prime}$, and let $E$ and $E^{\prime}$ be the corresponding exceptional divisors. Let $H^{\prime}, W, W^{\prime}$ be the strict transforms of $H, V, V^{\prime}$, respectively, and let $p^{\prime}:=p \circ g, q^{\prime}:=q \circ g: S \rightarrow \mathbb{P}^{1}$ be the composition morphisms. Then, we have

$$
-g^{\star} H_{0}=g^{\star}\left(K_{S_{0}}+D_{0}\right)=H^{\prime}+E+E^{\prime}=K_{S}+W+E+W^{\prime}+E^{\prime}+H .
$$

If we define the divisor $D$ by $D:=W+E+W^{\prime}+E^{\prime}+H^{\prime}$, then the above formula shows that $-\left(K_{S}+D\right)$ is nef. However, the sRC quotient of $(S, D)$ coincides with $p^{\prime}:(S, D) \rightarrow\left(\mathbb{P}^{1}, D_{\mathbb{P}^{1}}\right)$, where $D_{\mathbb{P}^{1}}$ is the orbifold divisor defined by the two points of the images of $V$ and $V^{\prime}$. Hence, $p^{\prime}:(S, D) \rightarrow\left(\mathbb{P}^{1}, D_{\mathbb{P}^{1}}\right)$ is obviously not a product.

\subsection{Higher-dimensional orbifolds with nef anti-canonical divisor}

One of the difficulties toward giving a proof of Conjecture 1.5 for the higher-dimensional case is the lack of a semistability theorem analogous to Theorem 3.4 because of the appearance of the orbifold divisor $D_{R}$. In this subsection, we prove some results in this direction.

THEOREM 5.11. Let $(X, D)$ be a smooth orbifold pair that is $l c$ and for which the anti-canonical divisor $-\left(K_{X}+D\right)$ is nef. Let $f:\left(X^{\prime}, D^{\prime}\right) \rightarrow\left(Z, D_{Z}\right)$ and $g:\left(X^{\prime}, D^{\prime}\right) \rightarrow(X, D)$ be a smooth neat representative of its $s R C$ quotient with the following commutative diagram:

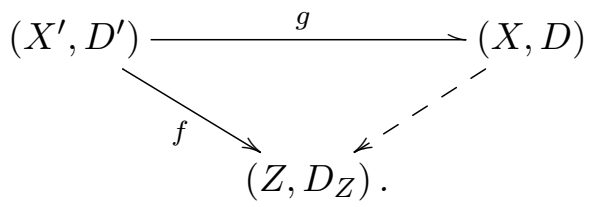

Then, we obtain $g_{\star}\left(f^{\star}\left(K_{Z}+D_{Z}\right)\right)=0$ and $g_{\star}(D(f, D))=0$; that is, these two effective divisors are $g$-exceptional. Here, $D(f, D)$ is the divisor defined by $D(f, D):=D(f)-D(f) / D_{Z}$ (see the proof below for the definitions of $D(f)$ and $\left.D(f) / D_{Z}\right)$.

Proof. First, note that $K_{Z}+D_{Z}$ is pseudo-effective by [Cam17, Theorem 1.5, Corollary 10.6] (see Theorem 1.4). We may assume that $g:\left(X^{\prime}, D^{\prime}\right) \rightarrow(X, D)$ is a birational morphism such that $\left(X^{\prime}, D^{\prime}\right)$ is a log smooth pair. We have the formula of canonical divisors

$$
g^{\star}\left(K_{X}+D\right)=K_{X^{\prime}}+D^{\prime}-E^{\bullet} \text { and } D^{\prime}=D^{\prime \prime}+\Delta^{\prime},
$$

where $D^{\prime \prime}$ is the strict transform of $D$ in $X^{\prime}$ and $\Delta^{\prime}$ and $E^{\bullet}$ are effective $g$-exceptional divisors with no common components. We may assume that $f:\left(X^{\prime}, D^{\prime}\right) \rightarrow Z$ is "neat" and its orbifold base $\left(Z, D_{Z}\right)$ is smooth. Note that $\left(X^{\prime}, D^{\prime}\right)$ is also lc because we are assuming that $(X, D)$ is lc.

The divisor $N^{\prime}$ defined by

$$
-N^{\prime}:=g^{\star}\left(K_{X}+D\right)=K_{X^{\prime}}+D^{\prime}-E^{\bullet}
$$




\section{F. Campana, J. Cao and S. Matsumura}

is nef by assumption. Thus, $N^{\prime}+\beta A^{\prime}$ is ample on $X^{\prime}$ for an arbitrary small rational number $\beta>0$ and a fixed polarization $A^{\prime}$ on $X^{\prime}$. We choose an effective $\mathbb{Q}$-divisor $B^{\prime}$ on $X^{\prime}$ with the following properties:

(1) The divisor $B^{\prime}$ is $\mathbb{Q}$-linearly equivalent to $N^{\prime}+\beta \cdot A^{\prime}$.

(2) The divisor $B^{\prime}$ is $f$-horizontal.

(3) The divisor $D^{+}:=D^{\prime}+B^{\prime}=D^{\prime \prime}+\Delta^{\prime}+B^{\prime}$ has snc support.

(4) The pair $\left(X^{\prime}, D^{+}\right)$is still lc.

Note that $K_{X^{\prime}}+D^{+}=K_{X^{\prime}}+D^{\prime}+B^{\prime} \equiv E^{\bullet}+\beta \cdot A^{\prime}$.

Both $f:\left(X^{\prime}, D^{+}\right) \rightarrow Z$ and $f:\left(X^{\prime}, D^{\prime}\right) \rightarrow Z$ have the same orbifold base $\left(Z, D_{Z}\right)$ because $B^{\prime}$ is $f$-horizontal. For the orbifold base $\left(Z, D_{Z}\right)$ of $f:\left(X^{\prime}, D^{+}\right) \rightarrow Z$, applying [Cam04, Theorem 4.11] and [CP19, Theorem 3.4] with a small adaptation shows that

$$
P_{\beta}:=K_{X^{\prime} / Z}+\left(D^{+}\right)^{\text {hor }}-D(f)-E^{\prime}
$$

is pseudo-effective, where $E^{\prime}$ is (not necessarily effective) $g$-exceptional. Here, $D(f)$ (respectively, $\left.D(f)_{/ D_{Z}}\right)$ is the effective $f$-vertical divisor defined by

$$
D(f)=\sum_{F \subset X^{\prime}}\left(t_{F}-1\right) \cdot F,
$$

where $F$ runs over all prime divisors of $X^{\prime}$ such that the image $f(F)$ by $f$ has codimension 1 (respectively, such that $f(F)$ has codimension 1 and is, moreover, contained in $D_{Z}$ ), and $t_{F}$ is the multiplicity of $F$ in $f^{\star} f(F)$.

Using Lemma 5.12 (see below), we can show that

$$
f^{\star} D_{Z}=D(f)_{/ D_{Z}}+\left(D^{\prime}\right)^{\text {vert }}-\Delta,
$$

for some effective divisor $\Delta$, and thus, from $\left(D^{+}\right)^{\text {vert }}=\left(D^{\prime}\right)^{\text {vert }}$, we obtain

$$
\begin{aligned}
P_{\beta}+E^{\prime} & =K_{X^{\prime} / Z}+\left(D^{+}\right)^{\text {hor }}-D(f) \\
& =K_{X^{\prime}}+D^{+}-f^{\star}\left(K_{Z}+D_{Z}\right)-\left[D(f)-D(f) / D_{Z}\right]-\Delta \\
& =K_{X^{\prime}}+D^{+}-f^{\star}\left(K_{Z}+D_{Z}\right)-D(f, D)-\Delta .
\end{aligned}
$$

Here, $D(f, D):=D(f)-D(f) / D_{Z}$ is the effective divisor consisting of the components of $D(f)$ whose image by $f$ is not contained in $D_{Z}$. Thus, we have

$$
P_{\beta}+f^{\star}\left(K_{Z}+D_{Z}\right) \equiv E^{\bullet}-E^{\prime}+\beta \cdot A^{\prime}-D(f, D)-\Delta .
$$

Then, letting $\beta \rightarrow 0^{+}$, we find that the pseudo-effective class $P$, which is the limit of the $P_{\beta}$, satisfies

$$
P+f^{\star}\left(K_{Z}+D_{Z}\right)=E^{\bullet}-E^{\prime}-D(f, D)-\Delta .
$$

Let $C \subset X$ be a general curve constructed by a complete intersection of ample divisors avoiding $g\left(E^{\prime}\right)$. We define the curve $C^{\prime}$ on $X^{\prime}$ as the inverse image of $C$ in $X^{\prime}$, so that $C^{\prime}$ does not intersect $E^{\bullet}$ and $E^{\prime}$. Then, we have

$$
0 \leqslant C^{\prime} \cdot f^{\star}\left(K_{Z}+D_{Z}\right) \leqslant C^{\prime} \cdot\left(P+f^{\star}\left(K_{Z}+D_{Z}\right)\right)=-C^{\prime} \cdot(D(f, D)+\Delta) \leqslant 0 .
$$

Note that the first inequality comes from the pseudo-effectivity of $K_{Z}+D_{Z}$. The curve $C^{\prime}$ satisfies $C^{\prime} \cdot M>0$ for any non- $g$-exceptional effective divisor $M$ on $X^{\prime}$, and thus we obtain the conclusion from the equalities $C^{\prime} \cdot f^{\star}\left(K_{Z}+D_{Z}\right)=C^{\prime} \cdot(D(f, D))=0$. 


\section{PROJECTIVE KLT PAIRS WITH NEF ANTI-CANONICAL DIVISOR}

Lemma 5.12. In the above notation, there exists an effective divisor $\Delta$ on $X^{\prime}$ such that $f^{\star} D_{Z}=$ $D(f) / D_{Z}+\left(D^{\prime}\right)^{\text {vert }}-\Delta$.

Proof. In this proof, $F$ denotes an irreducible divisor of $X^{\prime}$ such that $G_{F}:=f(F)$ is a component of $D_{Z}$. Then, up to some $f$-exceptional (hence, $g$-exceptional) divisors, we have

$$
\left(D^{\prime}\right)^{\text {vert }}=\sum_{F \subset X^{\prime}}\left(1-\frac{1}{m_{F}}\right) F,
$$

where $m_{F}$ is the multiplicity of $F$ in $D$. Additionally, when we write the multiplicity of $G_{F}$ in $D_{Z}$ as $m_{G_{F}}$, we have

$$
f^{\star} D_{Z}=\sum_{F \subset X^{\prime}} t_{F}\left(1-\frac{1}{m_{G_{F}}}\right) F .
$$

Thus, we can write

$$
t_{F}\left(1-\frac{1}{m_{G_{F}}}\right)=\left(t_{F}-1\right)+\left(1-\frac{1}{m_{F}}\right)-\left(\frac{t_{F}}{m_{G_{F}}}-\frac{1}{m_{F}}\right) .
$$

Further, by definition, we have $m_{G_{F}} \leqslant t_{F} \cdot m_{F}$, and so $\left(t_{F} / m_{G_{F}}-1 / m_{F}\right) \geqslant 0$. If we define the effective divisor $\Delta$ by

$$
\Delta:=\sum_{F \subset X^{\prime}}\left(\frac{t_{F}}{m_{G_{F}}}-\frac{1}{m_{F}}\right) F,
$$

then the above equality gives the conclusion $f^{\star} D_{Z}=D(f) / D_{Z}+\left(D^{\prime}\right)^{\text {vert }}-\Delta$.

Remark 5.13. The proof applies more generally when the pair $(X, D)$ is lc and not necessarily smooth. The proof does not require the notion of sRC or of the sRC quotient, only the fact that $K_{Z}+D_{Z}$ is pseudo-effective (although without these notions, we do not have any geometric description of the situations to which the result applies).

COROLlary 5.14. We retain the above notation. Then,

- if $B \subset Z$ is an irreducible divisor not contained in $D_{Z}$, any nonreduced component of $f^{\star} B$ is $g$-exceptional;

- if $G$ is a component of $D_{Z}$ and $F$ is an irreducible component of $f^{\star} G$ that is not $g$-exceptional, then $t_{F} \cdot m_{F}=m_{G}$.

More precisely, we obtain

$$
f^{\star} G=E+A+m_{G} \cdot B+C,
$$

where $E$ is the $g$-exceptional part of $f^{\star} G$, the divisor $A$ is reduced and contained in $D$ with all of its components of $D$-multiplicity $m_{G}$, while $B$ has no component in $D$, and $C$ consists of nonreduced components of $f^{\star} G$ contained in $D$.

Proof. Note that $A$ is not empty according to [GHS03]. The first assertion follows directly from Theorem 5.11. The second follows from its proof and the fact that $\left(C^{\prime} \cdot \Delta\right)=0$. The final assertion comes from the fact that if $F$ is not $g$-exceptional, we have $t_{F} \cdot m_{F}=m_{G}>1$. Thus, if $F$ is not in $D$, we have $m_{F}=1$, and thus $t_{F}=m_{G}$. If $F$ is reduced, $t_{F}=1$, and thus $m_{F}=m_{G}$.

Remark 5.15. If $G$ is a component of $D_{Z}$, and if $F$ is an irreducible component of $f^{\star} G$ and not $f$-exceptional, then $t_{F} \cdot m_{F} \geqslant m_{G}>1$. Hence, if $m_{F}=1$ (that is, $F$ is not contained in $D^{\prime}$ ), then $t_{F} \geqslant m_{G}>1$, and this may occur when $F$ is not $g$-exceptional. Otherwise, the divisor $F$ is a component of $\left(D^{\prime}\right)^{\text {vert }}$. 


\section{F. Campana, J. Cao and S. Matsumura}

\section{ACKNOWLEDGEMENTS}

The third author would like to thank Professor Yoshinori Gongyo for fruitful discussions on the work reported in [EG19]. He would also like to express his thanks to the members of the Institut de Mathématiques de Jussieu - Paris Rive gauche for their hospitality during his stay there.

\section{REFERENCES}

BCHM10 C. Birkar, P. Cascini, C. D. Hacon, and J. M'Kernan, Existence of minimal models for varieties of log general type, J. Amer. Math. Soc. 23 (2010), no. 2, 405-4688; doi:10.1090/S0894-034709-00649-3.

BDPP13 S. Boucksom, J.-P. Demailly, M. Păun, and T. Peternell, The pseudo-effective cone of a compact Kähler manifold and varieties of negative Kodaira dimension, J. Algebraic Geom. 22 (2013), no. 2, 201-248; doi:S1056-3911-2012-00574-8.

Bea83 A. Beauville, Variétés Kähleriennes dont la première classe de Chern est nulle, J. Differential Geom. 18 (1983), no. 4, 755-782; doi:10.4310/jdg/1214438181.

Ber09 B. Berndtsson, Curvature of vector bundles associated to holomorphic fibrations, Ann. of Math. 169 (2009), no. 2, 531-560; doi:10.4007/annals.2009.169.531.

BP08 B. Berndtsson and M. Păun, Bergman kernels and the pseudoeffectivity of relative canonical bundles, Duke Math. J. 145 (2008), no. 2, 341-378; doi:10.1215/00127094-2008-054.

BS94 S. Bando and Y.-T. Siu, Stable sheaves and Einstein-Hermitian metrics, in Geometry and Analysis on Complex Manifolds (World Sci. Publ., River Edge, NJ, 1994), 39-50; doi:10.1142/ 9789814350112_0002.

Cam92 F. Campana, Connexité rationnelle des variétés de Fano, Ann. Sci. École Norm. Sup. (4) 25 (1992), no. 5, 539-545; doi:10.24033/asens.1658.

Cam04_, Orbifolds, special varieties and classification theory, Ann. Inst. Fourier (Grenoble) 54 (2004), no. 3, 499-630; doi:10.5802/aif.2027.

Cam17_, Orbifold slope rational connectedness, 2017, arXiv:1607.07829v2.

Cao13 J. Cao, Théorèmes d'annulation et théorèmes de structure sur les variétés kähleriennes compactes, Ph.D. thesis, Université de Grenoble, 2013, available at https://tel.archivesouvertes.fr/tel-00919536.

Cao19 Albanese maps of projective manifolds with nef anticanonical bundles, Ann. Sci. Éc. Norm. Supér. (4) $\mathbf{5 2}$ (2019), no. 5, 1137-1154; doi:10.24033/asens.240.

CDM17 J. Cao, J.-P. Demailly, and S. Matsumura, A general extension theorem for cohomology classes on non reduced analytic subspaces, Sci. China Math. 60 (2017), no. 6, 949-962; doi:10.1007/ s11425-017-9066-0.

CDP15 F. Campana, J.-P. Demailly, and T. Peternell, Rationally connected manifolds and semipositivity of the Ricci curvature, in Recent Advances in Algebraic Geometry, London Math. Soc. Lecture Note Ser., vol. 417 (Cambridge Univ. Press, Cambridge, 2015), 71-91.

CF90 F. Campana and H. Flenner, A characterization of ample vector bundles on a curve, Math. Ann. 287 (1990), no. 4, 571-575; doi:10.1007/BF01446914.

CH19 J. Cao and A. Höring, A decomposition theorem for projective manifolds with nef anticanonical bundle, J. Algebraic Geom. 28 (2019), no. 3, 567-597; doi:10.1090/jag/715.

CP91 F. Campana and T. Peternell, Projective manifolds whose tangent bundles are numerically effective, Math. Ann. 289 (1991), no. 1, 169-187; doi:10.1007/BF01446566.

CP17 J. Cao and M. Păun, Kodaira dimension of algebraic fiber spaces over abelian varieties, Invent. Math. 207 (2017), no. 1, 345-387; doi:10.1007/s00222-016-0672-6. 


\section{PROJECTIVE KLT PAIRS WITH NEF ANTI-CANONICAL DIVISOR}

CP19 F. Campana and M. Păun, Foliations with positive slopes and birational stability of orbifold cotangent bundles, Publ. Math. Inst. Hautes Études Sci. 129 (2019), 1-49; doi:10.1007/ s10240-019-00105-w.

DPS93 J.-P. Demailly, T. Peternell, and M. Schneider, Kähler manifolds with numerically effective Ricci class, Compos. Math. 89 (1993), no. 2, 217-240, available at http://www.numdam.org/ item?id=CM_1993_-89_2_217_0.

DPS94_, Compact complex manifolds with numerically effective tangent bundles, J. Algebraic Geom. 3 (1994), no. 2, 295-345.

DPS96_ Compact Kähler manifolds with Hermitian semipositive anticanonical bundle, Compos. Math. 101 (1996), no. 2, 217-224, available at http://www.numdam.org/item?id=CM_1996_ _101_2_217_0.

EG19 S. Ejiri and Y. Gongyo, Nef anti-canonical divisors and rationally connected fibrations, Compos. Math. 155 (2019), no. 7, 1444-1456; doi:10.1112/s0010437×19007383.

EIM20 S. Ejiri, M. Iwai, and S. Matsumura, On asymptotic base loci of relative anti-canonical divisors of algebraic fiber spaces, 2020, arXiv:2005.04566.

GHS03 T. Graber, J. Harris, and J. Starr, Families of rationally connected varieties, J. Amer. Math. Soc. 16 (2003), no. 1, 57-67; doi:10.1090/S0894-0347-02-00402-2.

GKP16 D. Greb, S. Kebekus, and T. Peternell, Étale fundamental groups of Kawamata log terminal spaces, flat sheaves, and quotients of abelian varieties, Duke Math. J. 165 (2016), no. 10, 1965-2004; doi:10.1215/00127094-3450859.

Har67 R. Hartshorne, Local cohomology: a seminar given by A. Grothendieck, Harvard University, Fall, 1961, Lecture Notes in Math., vol. 41 (Springer-Verlag, Berlin - New York, 1967); doi: 10.1007/BFb0073971.

Har80 , Stable reflexive sheaves, Math. Ann. 254 (1980), no. 2, 121-176; doi:10.1007/ BF01467074.

HIM21 G. Hosono, M. Iwai, and S. Matsumura, On projective manifolds with pseudo-effective tangent bundle, J. Inst. Math. Jussieu, published online on 25 January 2021, doi:10.1017/ S1474748020000754, to appear in print.

HM07 C. D. Hacon and J. M'Kernan, On Shokurov's rational connectedness conjecture, Duke Math. J. 138 (2007), no. 1, 119-136; doi:10.1215/S0012-7094-07-13813-4.

Hör07 A. Höring, Uniruled varieties with split tangent bundle, Math. Z. 256 (2007), no. 3, 465-479; doi:10.1007/s00209-006-0072-5.

HP19 A. Höring and T. Peternell, Algebraic integrability of foliations with numerically trivial canonical bundle, Invent. Math. 216 (2019), no. 2, 395-419; doi:10.1007/s00222-018-00853-2.

HPS18 C. Hacon, M. Popa, and C. Schnell, Algebraic fiber spaces over abelian varieties: around a recent theorem by Cao and Păun, in Local and Global Methods in Algebraic Geometry, Contemp. Math., vol. 712 (Amer. Math. Soc., Providence, RI, 2018), 143-195; doi:10.1090/conm/712/ 14346.

KMM92 J. Kollár, Y. Miyaoka, and S. Mori, Rationally connected varieties, J. Algebraic Geom. 1 (1992), no. 3, 429-448.

Pău97 M. Păun, Sur le groupe fondamental des variétés kählériennes compactes à classe de Ricci numériquement effective, C. R. Acad. Sci. Paris Sér. I Math. 324 (1997), no. 11, 1249-1254; doi:10.1016/S0764-4442(99) 80408-X.

PT18 M. Păun and S. Takayama, Positivity of twisted relative pluricanonical bundles and their direct images, J. Algebraic Geom. 27 (2018), no. 2, 211-272; doi:10.1090/jag/702.

Wan20 J. Wang, Structure of projective varieties with nef anticanonical divisor: the case of log terminal singularities, 2020, arXiv:2005.05782.

Wu20 X. Wu, Pseudo-effective and numerically flat reflexive sheaves, 2020, arXiv:2004.14676. 


\section{F. Campana, J. Cao and S. Matsumura}

Zha96 Q. Zhang, On projective manifolds with nef anticanonical bundles, J. reine angew. Math. 478 (1996), 57-60; doi:10.1515/crll.1996.478.57.

Zha05_ On projective varieties with nef anticanonical divisors, Math. Ann. 332 (2005), no. 3, 697-703; doi:10.1007/s00208-005-0649-z.

Frédéric Campana frederic.campana@univ-lorraine.fr

Université de Lorraine, Institut de Mathématiques Élie Cartan, B.P. 70239, 54506 Vandoeuvreles-Nancy Cedex, France

Junyan Cao junyan.cao@unice.fr

Université Côte d'Azur, Laboratoire de Mathématiques J.A. Dieudonné, Parc Valrose, 06108 Nice cedex 02, France

Shin-ichi Matsumura mshinichi-math@tohoku.ac.jp mshinichi0@gmail.com

Mathematical Institute, Tohoku University, 6-3, Aramaki Aza-Aoba, Aoba-ku, Sendai 980-8578, Japan 